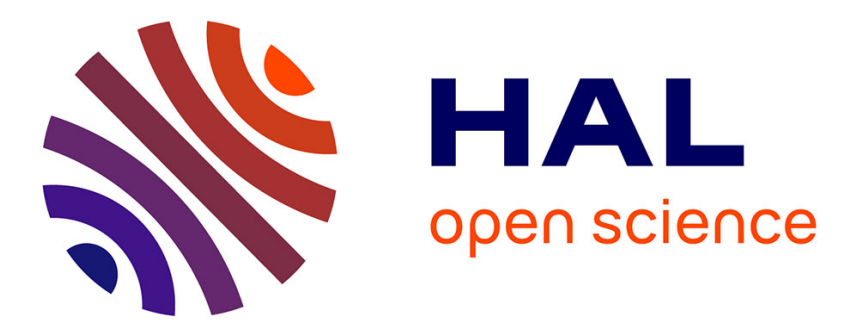

\title{
Infrared radiation applied to polymer processes
}

Yannick Le Maoult, Fabrice Schmidt

\section{To cite this version:}

Yannick Le Maoult, Fabrice Schmidt. Infrared radiation applied to polymer processes. Extrait de: Heat transfer in polymer composite materials (chap. 13) / sous la dir. de N. BOYARD, John Wiley; ISTE, p.385-423, 2016, Mechanical engineering and solid mechanics series, 978-1-84821-761-4. 10.1002/9781119116288.ch13 . hal-01725430

\section{HAL Id: hal-01725430 https://imt-mines-albi.hal.science/hal-01725430}

Submitted on 16 Apr 2019

HAL is a multi-disciplinary open access archive for the deposit and dissemination of scientific research documents, whether they are published or not. The documents may come from teaching and research institutions in France or abroad, or from public or private research centers.
L'archive ouverte pluridisciplinaire HAL, est destinée au dépôt et à la diffusion de documents scientifiques de niveau recherche, publiés ou non, émanant des établissements d'enseignement et de recherche français ou étrangers, des laboratoires publics ou privés. 


\section{Infrared Radiation Applied to Polymer Processes}

The infrared heating of polymers is often a crucial step during polymer processing, because radiative heat transfer can be very efficient in comparison to conductive or convective heat transfer. Indeed, the thermal conductivity of polymers is really low $(0.1 \leq \mathrm{k} \leq 0.6 \mathrm{~W} / \mathrm{m} / \mathrm{K})$ and consequently optimizing the heating and/or cooling steps remains a challenge. The results presented in this chapter are based on intensive research work, conducted at the ICA Institute over the past 10 years.

After a brief introduction, the authors present basic concepts dealing with radiative properties and more particularly infrared. The main radiative properties (emissivity, reflectivity, absorptivity, etc.) both for emitters and polymers are measured and analyzed. In addition, different methods to determine relevant parameters, such as filament lamp temperatures, are detailed. The development of accurate surface temperature measurements using an infrared camera is subsequently described. This powerful non-contact measurement device can also be applied for estimating heat transfer coefficients as well as anisotropic thermal conductivities of composites.

The infrared heating modeling of polymer preforms, typically used in the stretch blow molding process, is then presented. The assumptions of a cold and non-scattering medium suitable for thermoplastic polymers allow us to use a simplified radiative transfer equation. For radiation modeling, the Beer-Lambert law together with the ray tracing method is well adapted to simulate heating of semi-transparent polymers. Different examples are then presented, dealing with injection blow molding of P.E.T. bottles and curing of composites made of epoxide thermoset resin.

Future work and perspectives conclude this short but complete description of infrared radiation of polymers.

Chapter written by Yannick LE MAOULT and Fabrice SCHMIDT. 


\subsection{Introduction}

Radiant energy has numerous familiar applications. A very common one is that of microwave heating, nowadays adopted for cooking at home. Another application is the laser, most known by the public for surgical applications. Halogen lamps are appreciated for home lighting, as well as automotive lighting systems, and the drying of paints in the automotive industry is also a well-known application. The focus of this chapter, however, is on infrared heating of polymers.

\subsubsection{Why use infrared heating for polymers?}

In the industrial context of polymer processing, radiant energy has gained increasing markets due to low thermal conductivity of most polymers $(0.1-0.6$ $\mathrm{W} / \mathrm{m} / \mathrm{K}$ ) [FRA 02]. Different energy transfer means are used in industrial manufacturing processes:

- ultraviolet (UV);

- infrared (IR);

- high-frequency dielectric heating (HF);

- microwave dielectric heating (MO or UHF);

- laser beam.

The main advantages of IR heating include [ADE 02, WIL 14]:

- direct energy transfer between the source and the product to be treated (can penetrate into polymers giving a volumetric heating effect);

- high-power density;

- radiation focusing;

- control flexibility (low thermal inertia);

- low investment cost;

- adaptable and expandable.

\subsubsection{Application of radiative transfers in polymer processing}

In Table 13.1, some applications of infrared heating are presented. Polymer processes such as injection stretch-blow molding (ISBM) [ROS 04] and 
thermoforming [THR 99] are commonly used for manufacturing packaging products (bottles, cups, etc.). Both need a preliminary and efficient IR heating step before forming. In addition, development of IR heating technologies has gained recent interest for curing thermoset composites [WIL 14, NAK 11] since it is necessary to optimize manufacturing time and costs, and to provide the performances of these composites. Repair operation on damaged composite structures, such as an airplane fuselage, is another area in which infrared technologies are displacing conventional heating mats and conductive methods [WIL 14].

In the case of the ISBM process for manufacturing polyethylene terephthalate (PET) bottles, a tube-shaped preform previously injected is heated above the glass transition temperature $\left(T_{g} \cong 80^{\circ} \mathrm{C}\right)$ before stretching and inflating inside a bottle mold. It is well known that initial temperature distribution of the preform will have a major impact on mechanical and structural properties of the final bottle [BOR 09].

\begin{tabular}{|c|c|c|}
\hline Industry & Activity & Elementary operation can be processed by $I R$ \\
\hline \multirow{3}{*}{ Automotive } & Body & Gluing, varnishing, painting, printing \\
\hline & Equipment & $\begin{array}{c}\text { Surface treatment, gluing, forming, molding, } \\
\text { embossing }\end{array}$ \\
\hline & & Flocking, varnishing, painting, printing \\
\hline \multirow{2}{*}{$\begin{array}{l}\text { Thermoplastics, } \\
\text { composites }\end{array}$} & Technical parts & $\begin{array}{l}\text { Thermoforming, injection blow molding, } \\
\text { gluing, welding, curing }\end{array}$ \\
\hline & Flooring & $\begin{array}{l}\text { Varnishing, coating, polymerization, } \\
\text { foaming }\end{array}$ \\
\hline \multirow{2}{*}{ Textile } & Finishing & $\begin{array}{l}\text { Drying, dehydration, polymerization, thermo- } \\
\text { fixing, coating }\end{array}$ \\
\hline & $\begin{array}{l}\text { Technical } \\
\text { textiles }\end{array}$ & $\begin{array}{l}\text { Drying, heat treatment, gluing, polymerization, } \\
\text { thermo-fixing, forming, molding, graining, } \\
\text { coating }\end{array}$ \\
\hline
\end{tabular}

Table 13.1. Some industrial applications of infrared heating [ADE 02] 
In addition, the rate of bottle manufacture can exceed 60,000 bottles per hour for a current ISBM machine. It is this high production rate and the production method that exposes the ISBM bottle manufacturing process as a high-energy consumption process with around half the total energy of a consumable bottle of water lending itself to manufacturing (Figure 13.1).

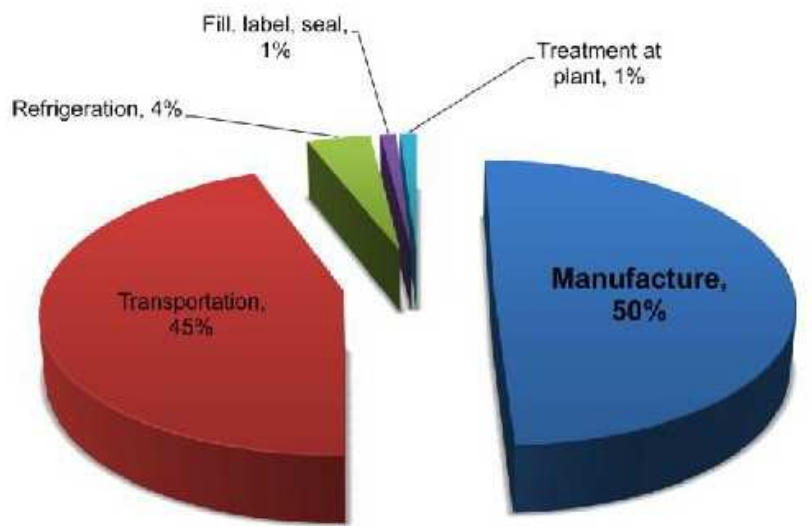

Figure 13.1. Energy required producing bottled water [NIX 15]. For a color version of the figure, see www.iste.co.uk/boyard/heat.zip

Optimizing temperature distribution while reducing energy consumption are major motivations to understand and model the interaction between IR radiation and the polymeric semi-products (preforms, sheets, plates, etc.).

\subsection{Infrared radiation characteristics}

Electromagnetic waves are in charge of heat transfer by radiation traveling at the speed of light $\left(c=2.998 \times 10^{8} \mathrm{~m} / \mathrm{s}\right.$ in vacuum $)$, which is called electromagnetic radiation. It is a kind of energy emitted as photons and exhibits as electromagnetic wave (Figure 13.2), which is characterized by wavelength $\lambda$ and frequency $v$ :

$$
\lambda=\frac{c}{v}
$$




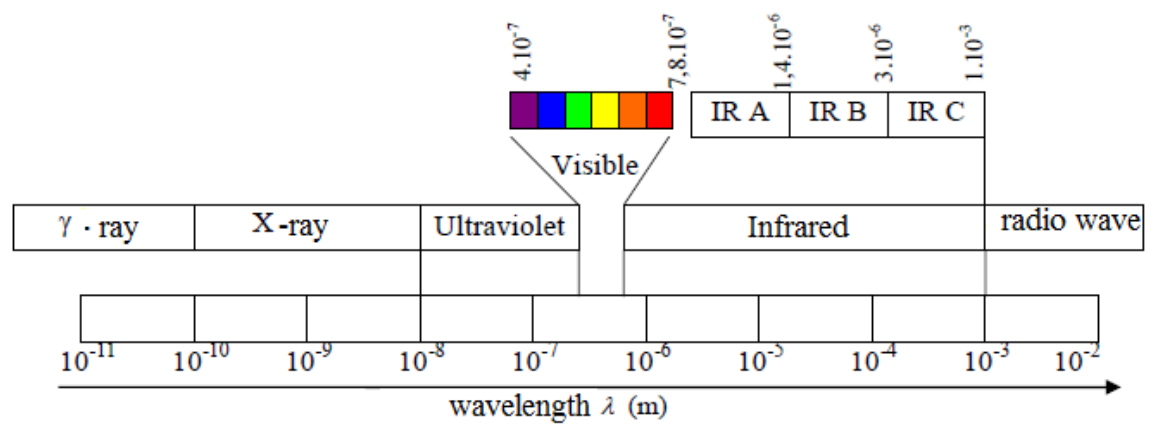

Figure 13.2. Electromagnetic wave spectrum [IEC 87]. For a color version of the figure, see www.iste.co.uk/boyard/heat.zip

According to most of the authors [MOD 03, SIE 02, MON 04], the characteristic infrared bandwidth is from $0.78-1,000 \mu \mathrm{m}$. Three main wavelength bands are used in sensing techniques or in the processing of materials: IR A [0.78-1.4 $\mu \mathrm{m}]$ corresponding to near-infrared, IR B [1.4-3 $\mu \mathrm{m}]$ corresponding to short-wavelength infrared (SW) and long-wavelength (LW) [8-15 $\mu \mathrm{m}]$.

\subsubsection{Radiative properties (basis and main definitions)}

The radiative heat flux $\Phi^{i}$ emitted by infrared lamps can be either reflected $\left(\rho_{\lambda}\right)$, absorbed $\left(\alpha_{\lambda}\right)$ or transmitted $\left(\tau_{\lambda}\right)$ by the polymer (Figure 13.3).

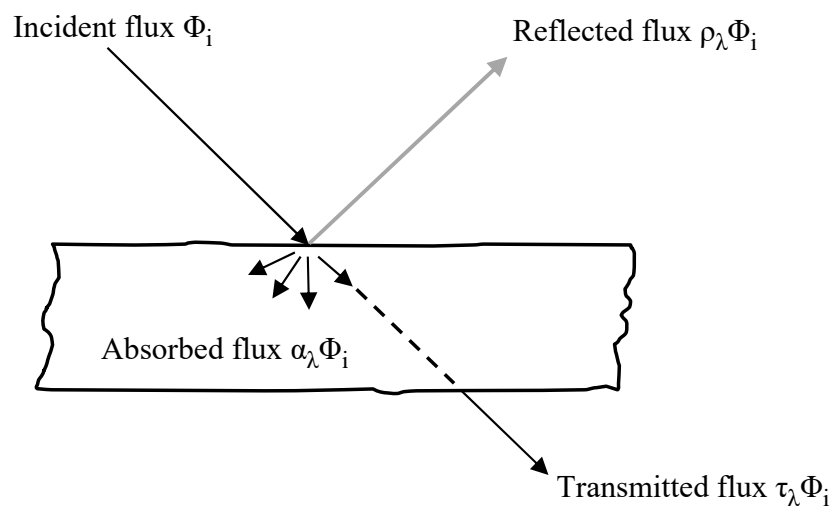

Figure 13.3. Radiative flux distribution 
Thus, Kirchhoff's first law simply expresses the energy balance:

$$
\alpha_{\lambda}+\tau_{\lambda}+\rho_{\lambda}=1
$$

In addition, using Kirchhoff's second law, we can assume the energy absorbed is equal to energy emitted:

$$
\varepsilon_{\lambda}=\alpha_{\lambda}=1-\tau_{\lambda}-\rho_{\lambda}
$$

where $\varepsilon_{\lambda}$ is the spectral emissivity coefficient. In addition, the reflection is related to the value of the surface roughness. Specular or diffuse reflection is characterized by a specific ratio given by $\frac{\sigma}{\lambda}$ where $\sigma$ is the root mean square (RMS) roughness of the surface and $\lambda$ is the wavelength of the incident radiation. If $\frac{\sigma}{\lambda}<<1$, then the surface is assumed to be specular; on the other hand, if $\frac{\sigma}{\lambda}>>1$, the surface is assumed to be a diffuse one. For most polymers parts considered here, the reflection is mainly diffuse. In addition, if the surface is assumed to be opaque, there is no radiation transmitted $\left(\tau_{\lambda}=0\right)$. The measurement of all these optical parameters is of prime importance in order to optically and spectrally characterize infrared heating of polymers and also to measure surface temperature distribution (mean emissivity coefficient is needed for infrared camera measurements).

\subsubsection{IR-spectrometer measurement (transmission and reflectivity)}

For the determination of the intrinsic transmittivity $\left(\tau_{\lambda}\right)$ and reflectivity $\left(\rho_{\lambda}\right)$ coefficients of semi-transparent polymers, transmission factor $\left(T_{\lambda}\right)$ and reflection factor $\left(R_{\lambda}\right)$ are measured using FT-IR spectrometer $(0.4-25 \mu \mathrm{m})$ together with an integrating sphere in the case of diffuse surface as sketched in Figure 13.4.

From the literature [SIE 02], expressions of transmission and reflection factors are well known:

$$
\left\{\begin{array}{c}
T_{\lambda}=\frac{\left(1-\rho_{\lambda}\right)^{2} \tau_{\lambda}}{1-\left(\rho_{\lambda} \tau_{\lambda}\right)^{2}} \\
R_{\lambda}=\rho_{\lambda}\left[1+\tau_{\lambda}^{2} \frac{\left(1-\rho_{\lambda}\right)^{2} \tau_{\lambda}^{2}}{1-\left(\rho_{\lambda} \tau_{\lambda}\right)^{2}}\right]
\end{array}\right.
$$




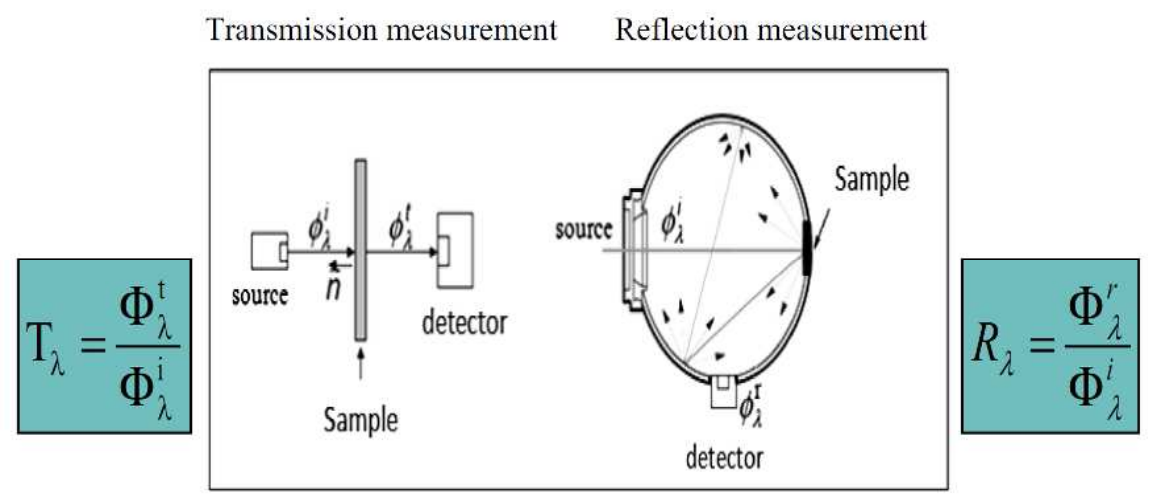

Figure 13.4. Radiative flux distribution [AKU 15]

Although a full resolution is possible using a numerical method [AKU 15], a simplified experimental approach, which has already been proven in several studies [BOR 09, MON 04], considers the following assumptions $\rho_{\lambda}\left\langle\left\langle 1\right.\right.$ and $\rho_{\lambda} \tau_{\lambda}\langle\langle 1$ for polymers material [SIE 02]:

$$
T_{\lambda} \cong \tau_{\lambda} \frac{1-\rho_{\lambda}}{1+\rho_{\lambda}} \Rightarrow-\ln T_{\lambda} \cong-\ln \tau_{\lambda}+\ln \frac{1+\rho_{\lambda}}{1-\rho_{\lambda}}
$$

If we consider non-scattering and cold medium assumptions (see section 13.3), transmittivity $\tau_{\lambda}$ is given according to the Beer-Lambert law:

$$
\tau_{\lambda}=e^{-\kappa_{\lambda} t}
$$

Equation [13.5] may then be simplified as follows:

$$
-\ln T_{\lambda} \cong \kappa_{\lambda} t+C_{\lambda}
$$

where $k_{\lambda}$ is the intrinsic absorption coefficient independent of the sample thickness $t$ and $C_{\lambda}$ is a value corresponding to calculation of the $-\operatorname{In} T_{\lambda}$ intercept of the experimental line. The $C_{\lambda}$ value permits us to calculate the reflectivity coefficient, in addition to reflection mode measurements that can be performed. 


\subsubsection{Example of transmission and reflection spectra for some polymers}

As mentioned before, PET is extensively used for the manufacture of thin-walled containers that are used for a multitude of applications, especially beverage containers. In order to achieve accurate transmission measurements, it is necessary to obtain thin samples at different thicknesses from amorphous PET injected-molded plaques or preforms. More details are described in [MON 01]. In Figure 13.5, the spectral transmission is plotted for two PET Tergal T74F9 $(\mathrm{IV}=0.74)$ samples $(0.24$ and $0.34 \mathrm{~mm}$ thicknesses).

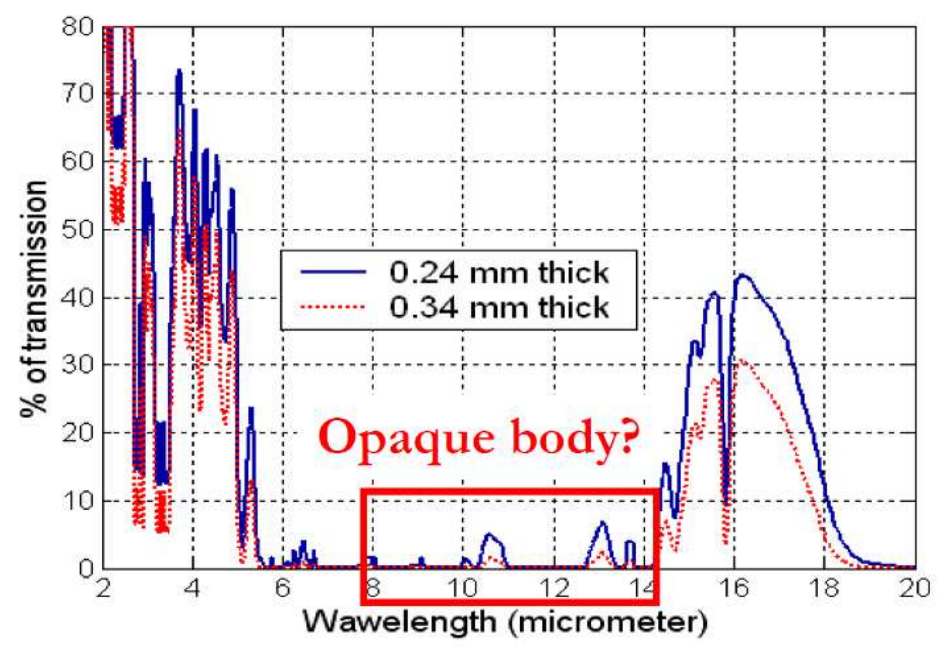

Figure 13.5. Spectral transmission coefficient of PET [BOR 07]. For a color version of the figure, see www.iste.co.uk/boyard/heat.zip

We note that for $\lambda \leq 7 \mu \mathrm{m}$, the transmission is very important. At the opposite, transmission is low in the spectral band $(8-14 \mu \mathrm{m})$. This means that PET can be considered as opaque in this specific wavelength band. This is not the case for all polymers. For example, HDPE is not opaque in this spectral band. In addition, reflection measurements for PET have been performed with $2 \mathrm{~mm}$ thick samples (Figure 13.6), confirming a low average value (approximately 7\%).

Further values for other polymers will be presented in the next section in correlation with lamp temperatures and the surface colors. 


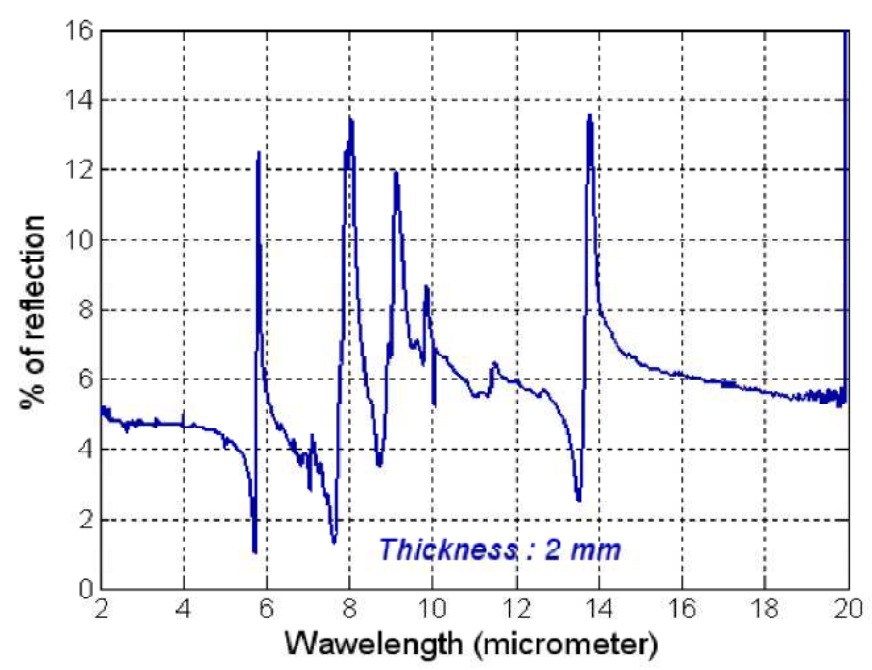

Figure 13.6. Spectral reflection coefficient of PET [BOR 07]

\subsubsection{Infrared emitters: characterization}

Infrared emitters vary mainly by the technology used, their geometry and temperature $T_{L}$. The role of temperature is predominant because it affects the maximum power and wavelength range associated with the element. The main problem is the choice of the wavelength range for radiative interactions between infrared lamps and polymers.

In addition, reflectors may change the spatial distribution of radiative flux. Other parameters, such as inertia during heating and cooling, can be critical depending on the type of application. As different technologies [KNI 96] exist on the market (metallic radiant tubes, quartz tubes, ceramics, vitrified ceramic radiant panels, catalytic and non-catalytic gas, etc.), we will focus here only on specific electrical emitters mainly used for ISBM and thermoforming processes: halogen, quartz tube and ceramic lamps.

The choice of emitters will be conditioned according to Wien's law giving the value of wavelength $\lambda_{m}$ for which spectral intensity $I_{\lambda}$ is maximal [SIE 02]:

$$
\lambda_{m}=\frac{2897.8}{T_{L}}\left(\mathrm{~T}_{\mathrm{L}} \text { in Kelvin }\right)
$$


Thus, the wavelength range associated with an emitter may be calculated by knowing that $95.6 \%$ of energy is between $0.5 \times \lambda_{m}$ and $5 \times \lambda_{m}$.

\subsubsection{Emitter temperature}

In Table 13.2, temperatures of typical emitters (halogen, quartz tube and ceramic lamps) are referenced as well as the related wavelength range. Note that for halogen lamps, most of the energy is emitted in the near-infrared (NIR) and shortwavelength infrared (SW).

\begin{tabular}{|c|c|c|c|}
\hline \multirow{2}{*}{ Quart tube } & Heater temperature $(\mathrm{K})$ & $\begin{array}{c}\text { Wavelength range } \\
\Delta \lambda(\mu \mathrm{m})\end{array}$ \\
\cline { 2 - 4 } & Quartz tube & 1,140 & $1.3 .-12.5$ \\
\cline { 2 - 4 } & Ceramic & 970 & $1.5-15$ \\
\cline { 2 - 4 } & Halogen lamp & 2,470 & $0.6-6$ \\
\hline
\end{tabular}

Table 13.2. Wavelength ranges for the different emitters [AND 05]

Using the previous measures of intrinsic spectral absorption coefficient (Figure 13.5) allows a mean penetration depth of radiation energy $\bar{e}(T)$ to be computed, which is the inverse of mean absorption coefficient $\bar{\kappa}(T)$ :

$$
\bar{e}(T)=\frac{1}{\bar{\kappa}(T)}=\frac{\int_{\Delta \lambda} I_{\lambda}^{0}(T) d \lambda}{\int_{\Delta \lambda} \kappa_{\lambda} I_{\lambda}^{0}(T) d \lambda}
$$

where $I_{\lambda}^{0}(T)$ is the blackbody spectral intensity given by Planck's law [MOD 03]. The spectral band $\Delta \lambda=[0.4,25] \mu \mathrm{m}$ corresponds to the typical range of the spectrometer Bruker Vertex 70 available in our laboratory. In Table 13.3, penetration depths are calculated for different thermoplastic polymers (ABS, PS and PP), natural, black (N) or white (B), considering temperatures of lamps (see Table 13.2). Indeed, most of the values are very low (a few tens of micrometer) and of the same order of magnitude for ceramic and quartz tube emitters. The highest 
value is for natural PP $(622 \mu \mathrm{m})$ together with halogen lamp. In comparison (Figure 13.5), transmission radiation is much more important for PET polymer and particularly for NIR.

\begin{tabular}{|c|c|c|c|c|c|c|c|c|}
\hline $\bar{e}(\mu \mathrm{m})$ & 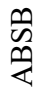 & 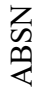 & $\mathscr{2}$ & $\stackrel{n}{\infty}$ & $\begin{array}{l}Z \\
\tilde{a}\end{array}$ & 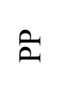 & $\frac{m}{2}$ & 文 \\
\hline Ceramic & 69 & 29 & 195 & 64 & 39 & 429 & 157 & 65 \\
\hline Quartz tube & 64 & 26 & 215 & 64 & 35 & 451 & 157 & 59 \\
\hline Halogen & 41 & 18 & 233 & 79 & 29 & 622 & 128 & 44 \\
\hline
\end{tabular}

Table 13.3. Mean penetration depths [AND 05]

The effect of color can be further highlighted by calculating the mean reflectivity coefficient $\bar{\rho}(T)$ on the same spectral band:

$$
\bar{\rho}(T)=\frac{\int_{\Delta \lambda} \rho_{\lambda} I_{\lambda}^{0}(T) d \lambda}{\int_{\Delta \lambda} I_{\lambda}^{0}(T) d \lambda}
$$

Most of the values are less than 10\%, as expected (section 13.2.1.2). But for white samples with halogen lamps (NIR and SW), the mean reflectivity coefficient may be more than $40 \%$. These results confirm that infrared heating using halogen lamps, will be affected by the color of the surface, particularly by white.

\begin{tabular}{|c|c|c|c|c|c|c|c|c|}
\hline $\bar{\rho}(T)(\%)$ & 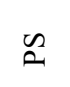 & $\stackrel{\mathscr{n}}{\tilde{2}}$ & $Z_{\mathscr{L}}$ & $\hat{2}$ & $\stackrel{m}{\stackrel{m}{a}}$ & 文 & $\begin{array}{l}\mathscr{V} \\
\tilde{\vartheta}\end{array}$ & $\begin{array}{l}Z \\
\text { Z } \\
\text { qu }\end{array}$ \\
\hline Ceramic & 2.9 & 3.9 & 2.7 & 3.9 & 4.1 & 3.5 & 4.6 & 4.1 \\
\hline Quartz tube & 6.3 & 10.0 & 3.6 & 7.9 & 10.2 & 3.9 & 11.4 & 4.9 \\
\hline Halogen & 19.8 & 41.5 & 6.3 & 19.0 & 41.3 & 5.2 & 45.6 & 6.5 \\
\hline
\end{tabular}

Table 13.4. Mean reflectivity coefficient [AND 05]

\subsubsection{Emission spectrum of halogen lamps}

Considering now the halogen lamp, a coiled tungsten filament is enclosed into a quartz tube filled with neutral gas. In order to calculate the power emitted by the 
lamp $P_{L}$, we need to know the spectral emissivity of the tungsten filament versus temperature $\varepsilon_{\lambda}^{F}\left(T_{F}\right)$. Using data issued from the literature [MON 01] and completing for $\lambda \geq 2.6 \mu \mathrm{m}$ using Hagen-Rubens law [SIE 02], allows an estimation of the spectral emissivity for pure tungsten (Figure 13.7). Indeed, we observe strong variations versus wavelength and temperature that prevents assuming this parameter constant. Thus, we can estimate the power emitted by the lamp as follows:

$$
P_{L}=\sigma_{S B} \bar{\varepsilon}^{F}\left(T_{F}\right) T_{F}^{4} S_{F}
$$

where $\sigma_{S B}$ is the Stefan-Boltzmann constant [SIE 02], $S_{F}$ is the area of filament, $T_{F}$ is the temperature of the filament and $\bar{\varepsilon}^{F}$ is the mean emissivity, defined following the same way as the mean reflectivity coefficient (equation [13.10]).

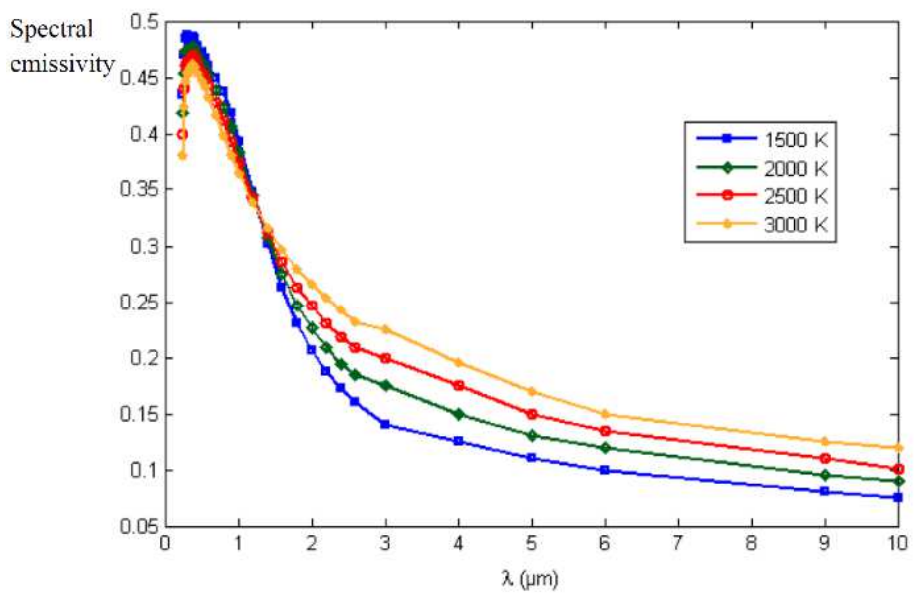

Figure 13.7. Spectral emissivity of pure tungsten [MON 01]. For a color version of the figure, see www.iste.co.uk/boyard/heat.zip

Of course, the filament is not made only of pure tungsten, but of an alloy. For $T_{F}=2470 \mathrm{~K}$ (i.e. the temperature of a halogen lamp), the mean emissivity is equal to 0.27 . In addition, the quartz tube also emits and contributes to $9 \%$ of total emitted power [MON 01].

\subsubsection{Directional emission of typical lamps used in forming processes}

An in-lab experimental set-up using a thermopile (for full details, see [MON 01] and [AND 05]) has been developed in order to evaluate directivity of each emitter 
and to provide data for computational methods described later in this chapter. In Figure 13.8, polar emissions of different heaters are plotted and compared to Lambertian source (isotropic emission).

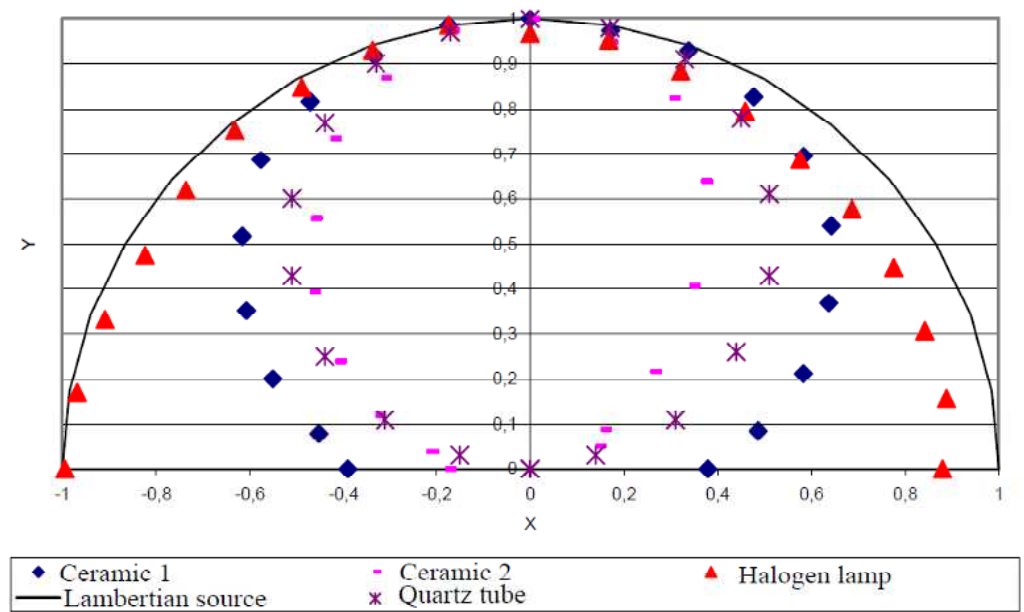

Figure 13.8. Polar emission of different heaters [AND 05]. For a color version of the figure, see www.iste.co.uk/boyard/heat.zip

We note that a "clear" halogen lamp (i.e. with no ceramic coating on the back surface of the quartz tube) is similar to a Lambertian source. This is not the case for all the other emitters.

\subsubsection{Infrared camera measurements}

One of the key points in polymer processing is the control of surface temperature of parts or preforms during the heating step and, as a consequence, the optimization of the volumic heat source inside the material to ensure the best efficiency of heating. Due to the high production rate needed for plastic bottles, as seen before, this step is mainly provided by IR lamps.

Some tests have been conducted by researchers to evaluate the ability of thermocouples as a temperature sensor on the surface or inside the polymer heated [BAR 94]. These authors have shown that the response of the embedded thermocouples in a semi-transparent material such as PET can be drastically affected by the IR radiation traveling through the material toward the thermoelectric 
junction. This leads to errors in the temperature measurements which can be very different from the true local temperature of the polymer. This drawback implies rigorous corrections (that means a specific model) of the measurement process. Moreover, the size of the thermocouple has to be chosen very carefully to avoid conduction into the wires. Such a method is not suited to quick experiments on industrial machines where processing time is a critical parameter.

For these reasons, non-contact temperature measurement has been tested in our approach. Some authors proposed in the past to use infrared pyrometers [HAI 94] and gave comparisons depending on spectral properties of the material observed. To be more complete, we have chosen temperature field measurement with an infrared camera instead of single spot analysis. In all these situations, we have to take into account a radiometric equation related to different parameters which are necessary to convert raw digital values obtained at the output of the camera into true temperatures. The radiometric equation is given by:

$$
D L^{0}\left(T_{e q}\right)=\tau_{a t m}\left(T_{a t m}\right)\left(\varepsilon_{p o l y m} D L^{0}\left(T_{p o l y m}\right)+\rho_{\text {polym }} D L^{0}\left(T_{e n v}\right)\right)+D L^{0}\left(T_{a t m}\right)
$$

- $D L^{0}\left(T_{e q}\right)$ corresponds to the digital level (DL) delivered by the camera, $T_{e q}$ is an apparent blackbody temperature;

- $\tau_{\text {atm }}$ represents the atmospheric transmission along the optical path between the camera and the polymer semi-product (preform, sheet, etc.);

- $D L^{0}\left(T_{p o l y m}\right)$ corresponds to the signal delivered by the camera when the target seen is a blackbody at the temperature of the polymer semi-product;

- $D L^{0}\left(T_{e n v}\right)$ is the signal related to an environment term which will be discussed later;

- $D L^{0}\left(T_{a t m}\right)$ is a contribution given by an equivalent blackbody level and corresponding to the atmospheric contribution for long distance measurement.

To obtain these different terms, we need to investigate a typical experimental situation encountered in our problem. This situation is depicted in Figure 13.9 and shows our in-lab blow molding machine including an infrared oven on the right side with six lamps (1,000 W each), a reflector behind (can be an aluminum plate or a specific white ceramic) and on the left side the preform and the mold dedicated to the blowing step of a 0.51 bottle after the heating step. 


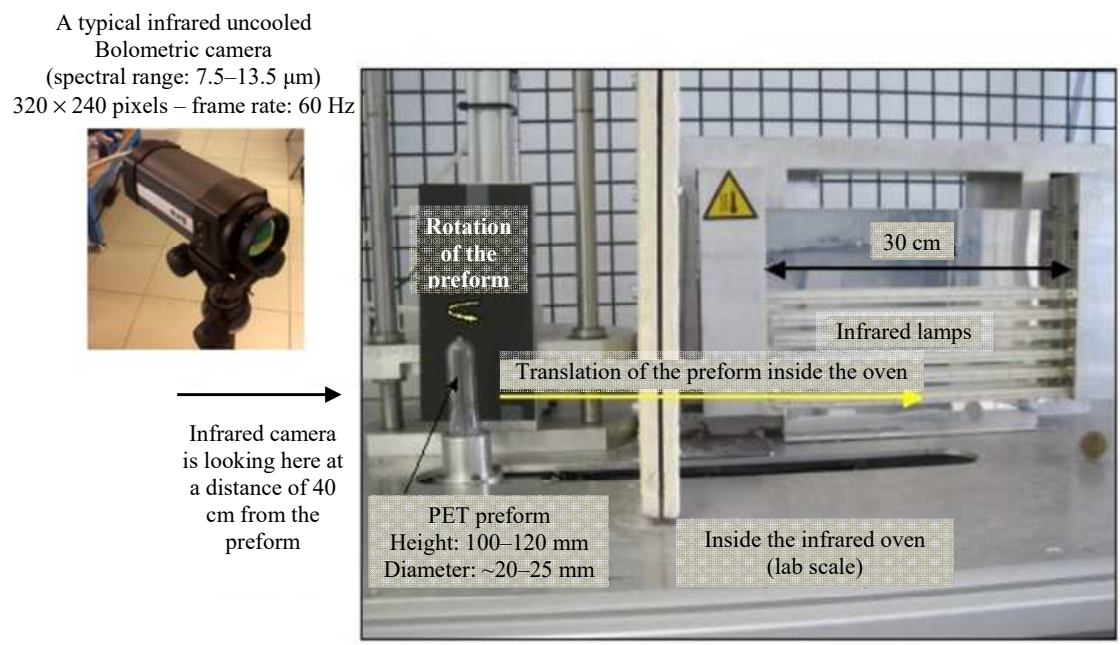

Figure 13.9. In-lab infrared oven

At first, it is important to choose the best spectral range for the camera to ensure that the measurement done corresponds to a surface temperature. With the help of equation [13.9] and choosing specific spectral ranges (short wave, medium wave and long wave), it is easy to calculate the penetration depth (see section 13.2.2.1). For example, if we consider PET, the results for e(T), discussed in [MON 04], are summarized in Table 13.5.

\begin{tabular}{|c|c|c|c|}
\hline $\mathrm{e}(\mathrm{T})$ spectral range & $1-2.5 \mu \mathrm{m}$ & $2.5-5 \mu \mathrm{m}$ & $5-20 \mu \mathrm{m}$ \\
\hline $\mathrm{T}=2,400 / 2,500 \mathrm{~K}$ & $3.2 \mathrm{~mm}$ & $0.4 \mathrm{~mm}$ & 0 \\
\hline $\mathrm{T}=400 \mathrm{~K}$ & $0.5 \mathrm{~mm}$ & $0.25 \mathrm{~mm}$ & 30. $10^{-3} \mathrm{~mm}$ \\
\hline
\end{tabular}

Table 13.5. Penetration depths for $T=2,400 / 2,500 \mathrm{~K}$ and $T=400 \mathrm{~K}$

It can be seen that the 5-20 $\mu \mathrm{m}$ wave range corresponds to the opaque region: the incident radiative flux coming from the lamps is considered as a boundary condition at the surface and regarding the emission of the preform, the flux received by an infrared sensor can be assumed as a surface emission (not a volume emission). 
For this reason, the best compromise here is to choose the 7-14 $\mu \mathrm{m}$ window available among the different ranges for infrared cameras. Most used windows are 7.5-13.5 $\mu \mathrm{m}$ or $8-12 \mu \mathrm{m}$ depending on the supplier of the camera, which also fits to the best atmospheric transmission window for the infrared [WOL 89].

Note also that for the lamp temperature and the short wave range, $\mathrm{e}(\mathrm{T})$ is equal to $3.2 \mathrm{~mm}$, a value greater than the real thickness of our preform $(3 \mathrm{~mm})$. This shows that for the maximum of emission of the lamp $(\cong 1.2 \mu \mathrm{m})$, the radiative energy contributes to a volumic heating of the preform (see section 13.2.2). As a conclusion, by choosing the 7-14 $\mu \mathrm{m}$ spectral range, a real surface temperature measurement can be performed. To ensure that this temperature is a true temperature, we now have to consider the other terms of [13.12]:

- $\tau_{\text {atm }}$ (atmospheric transmission): for the spectral range 7-14 $\mu \mathrm{m}$, a simple test done with a blackbody shows that there is no significant attenuation of the signal received by the camera when the blackbody is moved from a very short distance up to several meters. As the preform is viewed at less than $0.5 \mathrm{~m}$, the $\tau_{a t m}$ parameter is assumed to be equal to 1 ;

- as a result of Table 13.5, PET is an opaque material in the 7-14 $\mu \mathrm{m}$ spectral region). Then, a reflectance measurement done on an FTIR spectrometer gives $\bar{\rho}=0.07$ as a mean value of $\rho_{\lambda}$ between 7 and $14 \mu \mathrm{m}$, this leads to $\bar{\varepsilon}=0.93$ for our PET Tergal T74F9 (IV=0.74) (this value can vary slightly according to the grade of PET used);

- contribution of the atmosphere in the emission $\left(D L^{0}\left(T_{a t m}\right)\right.$ term): due to the very short distance between the camera and the preform and the spectral range used in our measurements, this term is assumed to be negligible;

$-D L^{0}\left(T_{\text {env }}\right)$ : this term requires special attention depending on the geometric configuration of the oven used. For an "open" oven: the environment term is related to a radiative contribution coming from the wall of the room where the oven is installed (a "room" temperature). Then, a measurement made with the infrared camera on a homogeneous screen beside the heating area, measurement made at room temperature (every part of the oven is in radiative equilibrium with the surroundings) gives the $D L^{0}\left(T_{\text {env }}\right)$ and in a second step $T_{\text {env }}$ by inverting the calibration curve $D L^{0}$. However, such a situation can correspond only to a simple lab situation. In a more realistic situation, the oven is composed of different walls and reflectors in front of the panel of lamps or ventilation system as depicted in Figure 13.10. 
(1)

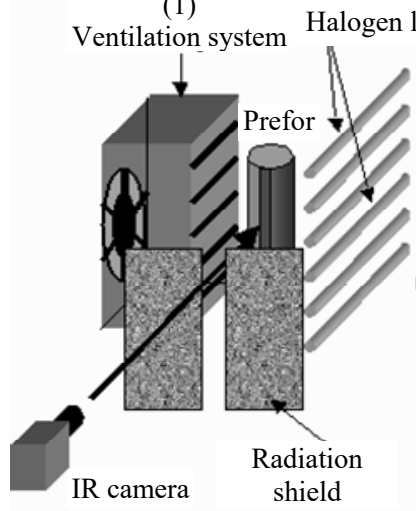

(a) The thermal scene
(2)

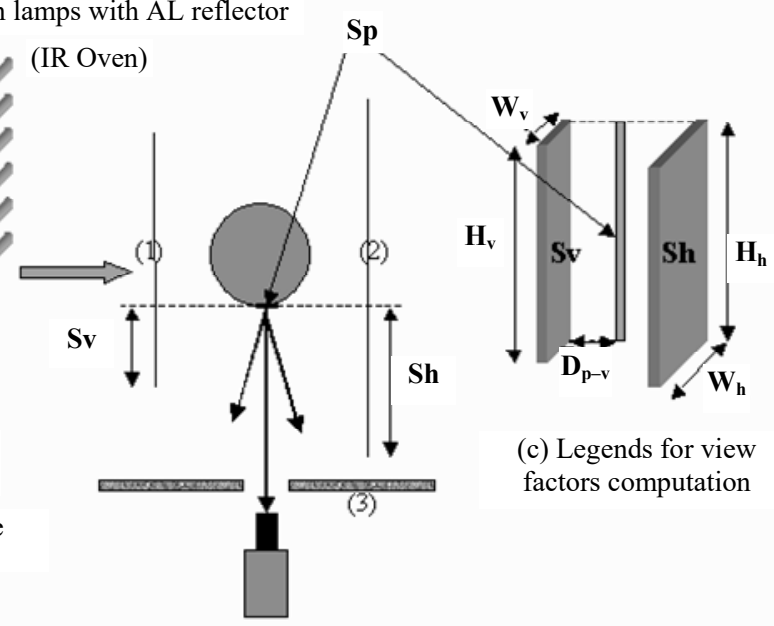

(b) Top view (not in scale)

Sp: selected area (vertical strip) on the preform for the temperature measurements

Sv: restricted ventilation support area visible by $\mathrm{Sp}$

Sh: restricted halogen lamps area visible by $\mathrm{Sp}$

Figure 13.10. A more realistic configuration (our system)

Each element, depending on its own temperature and optical properties, can contribute to the energy measured by the infrared camera on a vertical strip $S_{p}$ on the front of the preform as shown in Figure 13.10 (the size of this vertical strip is taken greater than a given number of pixels depending on the spatial resolution of the camera used). The reflectors and grid of the cooling fan are considered flat surfaces (i.e. here $S_{v}$ and $S_{k}$ ) for which optical properties such as reflectivity have been analyzed with an infrared spectrometer, and temperatures have been recorded with a specific thermocouple fixed on the inner part of each plate. With these data, the energy exchanged, in a first approach, between the ventilation system and the preform and between the halogen lamps with a back surface plane has been obtained via a view factors computation (e.g. plate to plate or strip to plate).

As in a radiosity method, we have then computed the sum of these last two terms incoming on the camera lens (i.e. irradiance $E_{s}$ in $\mathrm{W} / \mathrm{m}^{2}$ ). Then, a numerical calibration curve has been provided involving $T_{b b}=f\left(E_{b}\right)$ where $E_{b}$ was the irradiance in $W / \mathrm{m}^{2}$ when the preform is assumed to be a perfect blackbody, for 
different levels of irradiance. The irradiance $E_{s}$ has also been plotted, with measured optical properties of the preform and the different components of our oven, for different levels of temperature of the preform reproducing the heating stage. Lastly, the two curves were compared to quantify the discrepancy induced by the effect of the walls of the oven. By considering that the computation of $E_{s}$ is another way to establish [13.12], we tested in the computations an equivalent environment temperature $T_{e n v}$ (global contribution of reflections on the preform).

This parametric study leads to $T_{e n v} \cong 25^{\circ} \mathrm{C}$, giving good agreement with the "blackbody curve": $\Delta T \cong 1^{\circ} \mathrm{C}$ around $120^{\circ} \mathrm{C}$. An experimental approach has also been tested: an image difference technique between the preform viewed by the infrared camera out of the oven and the preform just as the entrance of the oven: the preform is still in equilibrium with the room temperature but the reflections of the new environment can be deduced; this other approach shows a $T_{e n v}$ more close to $27 / 28^{\circ} \mathrm{C}$ but the difference between computation and experiment had no significant effect on the non-contact measurement done on the preform in the oven $\left(1\right.$ or $2^{\circ} \mathrm{C}$ around $120^{\circ} \mathrm{C}$ ). As a conclusion, a temperature map on the PET preform was successfully achieved inside the infrared oven, generally considered as difficult conditions. An example of such a map is given in Figure 13.11.

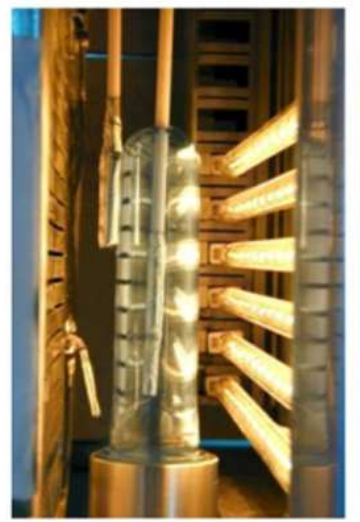

PET preform inside the lab infrared oven

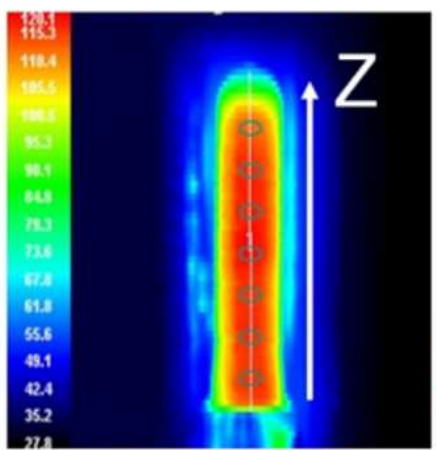

Infrared thermogram showing markers on the height of the preform (temperature spots)

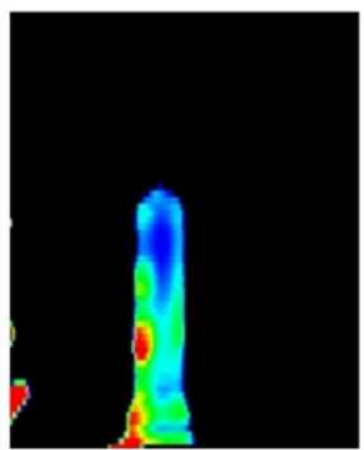

Non-homogeneous heating: IR thermography as an optimization tool for the process

Figure 13.11. Quantitative infrared thermography inside the lab infrared oven. For a color version of the figure, see www.iste.co.uk/boyard/heat.zip 
This section has shown the different steps to use an infrared camera, not only for thermal imaging but also to achieve quantitative infrared thermography. As mentioned before, these measurements had been used to obtain temperature spots of profiles along the height of the preform helping to adjust the oven set-up regarding the variation of the temperature on the PET.

Other applications, such as validation of lamp temperatures via infrared thermography measurements on quartz tubes (indirect validation of filament temperature) [MAO 05] or heat transfer convection coefficient along the height ( $\mathrm{Z}$ ) of the preform [MON 04] helping to complete the dataset needed for a complete simulation of the heating step, are described in [MON 01].

\subsection{Modeling of infrared radiation}

As previously shown, characterizing and measuring temperature of infrared polymers is essential before developing radiation simulations. In section 13.2.2, we highlighted that thermoplastics can be opaque or semi-transparent, depending on the type of emitters. In addition, in some kind of composite materials, due to the presence of fibers reinforcement and particularly carbon fibers, it is the same for thermoplastic or thermoset composites [NAK 11]. In the following section, we will consider both opaque and semi-transparent cases.

\subsubsection{Opaque medium: surface to surface methods}

Entire surfaces (tungsten filament, reflectors, composites or polymers, etc.) are assumed opaque in the infrared bandwidth, and behave like a Lambertian source (isotropic emission). In addition, only integrated optical properties should be considered. An ambient medium (for example, air) is assumed to be a nonparticipative radiative medium.

Thus, surface to surface methods could be applied in order to account for radiation interactions. Then, radiation heat transfer between opaque surfaces is based upon radiosity which is defined as the total radiative flux leaving a surface. The radiosity method subdivides a scene into different areas acting as Lambertian emitters and subsequently determines a condition in which the radiative transfer between the areas leads to an energetic equilibrium [SIE 02]. The fraction of radiative energy absorbed by the composite (or opaque polymer) may be calculated via view factor computations. 
Different analytical approaches exist for simple view factor calculations [SIE 02]. However, most of the time, numerical methods are necessary to account for complex geometries. The main advantage of this approach is the reduction of computer time in comparison to more general methods such as ray tracing and Monte Carlo. Figure 13.12(a) shows the geometry of a 3D composite part heated by one halogen heater. The global error between heat fluxes computed using hemicube numerical method [COH 85] and ray tracing method (Figure 13.12(b)) is less than $10 \%$. In addition, CPU time is reduced approximately by 7 (Intel Core 2Duo $2.1 \mathrm{Ghz} 2.99 \mathrm{Go})$.
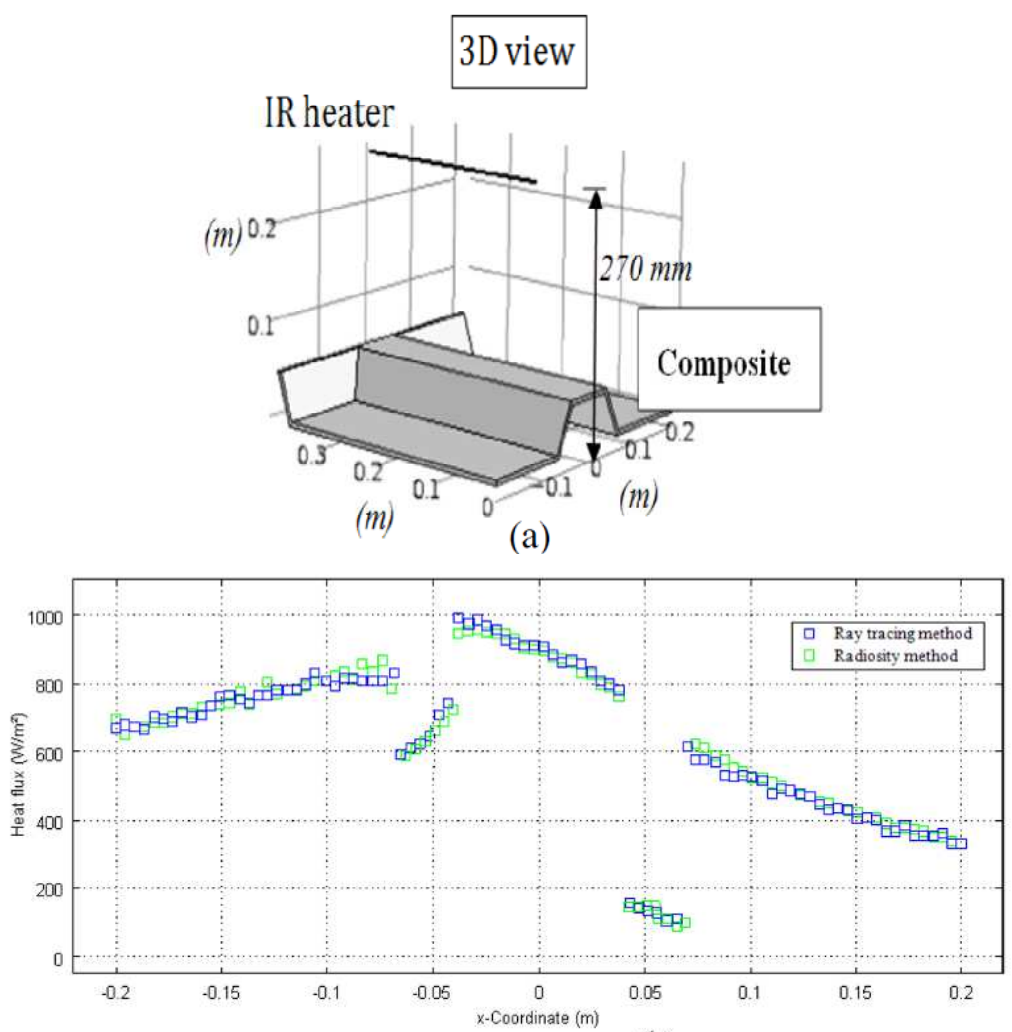

(b)

Figure 13.12. Heat flux along width [NAK 13]. For a color version of the figure, see www.iste.co.uk/boyard/heat.zip

In order to develop optimization procedures for improving surface temperature distributions, computation times for solving a radiative transfer problem should be 
greatly minimized. It should be noted that this simplified approach is not useable for infrared heating of semi-transparent polymers or thermoplastic composites, where absorption or scattering has to be accounted for.

\subsubsection{Semi-transparent medium}

The propagation of radiation through a semi-transparent medium is affected by absorption, emission and scattering processes. The radiative transfer equation describes the interaction of photons inside the matter, that is to say: losses of energy related to absorption, gains energy by emission and scattering.

\subsubsection{Radiative transfer equation}

The fundamental quantity which describes a field of radiation is the so-called spectral intensity $I_{\lambda}(\vec{s}, \vec{\Omega})$ depending on the position vector $\vec{s}$ and the direction vector $\vec{\Omega}$. Thus, the differential form of the equation for radiative transfer is as follows:

$$
\frac{d I_{\lambda}(\vec{s}, \vec{\Omega})}{d s}+\underbrace{\left(\kappa_{\lambda}+D_{\lambda}\right) I_{\lambda}(\vec{s}, \vec{\Omega})}_{\text {absorption }}=\underbrace{\kappa_{\lambda} I_{\lambda}^{0}(T(s))}_{\text {emission }}+\underbrace{\frac{D_{\lambda}}{4 \pi} \int_{0}^{4 \pi} \psi\left(\vec{\Omega}, \vec{\Omega}^{\prime}\right) I_{\lambda}\left(\vec{s}, \vec{\Omega}^{\prime}\right) d \Omega^{\prime}}_{\text {scattering }}[13.13]
$$

where $D_{\lambda}$ is the scattering coefficient, $\psi$ is the phase function, $\Omega^{\prime}$ is the solid angle and $\vec{\Omega}^{\prime}$ is the scattering direction vector.

Radiation scattering may occur during infrared heating of semi-transparent thermoplastics for which optical properties are influenced by the crystalline microstructure (morphology and/or degree of crystallinity). Another interesting case is the radiation heating of a semi-transparent thermoplastic composite (Figure 13.13) using a laser beam. The difference between refractive indices of the polycarbonate matrix and the glass fibers will induce light scattering and attenuation of the intensity. In this case, accurate measurement of the scattering coefficient $D_{\lambda}$ will be crucial.

\subsubsection{Cold and non-scattering material assumption: Beer-Lambert law}

For many thermoplastic polymers, it is possible to neglect scattering during infrared heating. This is typically the case for PET during the heat conditioning step of the ISBM process. Degree of crystallization after injection molding of PET preforms is less than $5 \%$ [MON 01]. This assumption is no more possible for ISBM 
of mineral filled PET where particles may aggregate with a size up to $0.5 \mu \mathrm{m}$ [BIL 12].

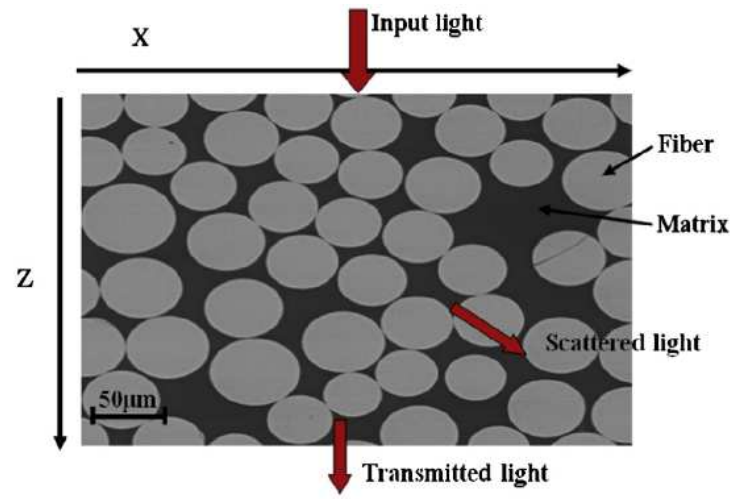

Figure 13.13. UD composite microstructure (polycarbonate matrix and $40 \%$ glass fibers) [AKU 15]

Another important assumption is the so-called "cold medium". In fact, selfemission of most of the heated polymers may be neglected during the heating step, considering that the polymer bulk temperature $\left(T_{P E T} \leq 400 \mathrm{~K}\right)$ is very low in comparison to the source temperature $\left(T_{F} \leq 2000 \mathrm{~K}\right)$ for tungsten filament). Therefore, the assumption of a cold material is convenient [ $\mathrm{SCH} 03$ ].

Using these assumptions greatly simplifies the radiative transfer equation and allows integrating easily:

$$
\frac{d I_{\lambda}(\vec{s}, \vec{\Omega})}{d s}=-\kappa_{\lambda} I_{\lambda}(\vec{s}, \vec{\Omega}) \Rightarrow I_{\lambda}(\vec{s}, \vec{\Omega})=I_{\lambda}\left(\vec{s}_{o}, \vec{\Omega}\right) e^{-\kappa_{\lambda}\left(s-s_{o}\right)}
$$

where $s_{o}$ can be, for example, the position vector at the polymer surface. We obtain a similar relation to Beer-Lambert law previously used in section 13.2.1.1.

For the case of a semi-transparent material, the heat balance equation may be written as follows:

$$
\rho c_{p} \frac{d T}{d t}=\vec{\nabla} \cdot(k \vec{\nabla} T)-\vec{\nabla} \cdot \vec{q}_{r}
$$


where $\rho$ is the polymer density, $c_{p}$ is the specific heat and $\vec{q}_{r}$ is the radiative flux. In this case, the radiative flux is simply approximated using the Beer-Lambert law [COS 11]:

$$
\vec{\nabla} \cdot \vec{q} \cong-\int_{0}^{\infty} \kappa_{\lambda} e^{-\kappa_{\lambda}\left(s-s_{o}\right)} \int_{0}^{4 \pi} I_{\lambda}\left(\vec{S}_{o}, \vec{\Omega}\right) d \Omega d \lambda=-\int_{0}^{\infty} \kappa_{\lambda} M_{\lambda}\left(\vec{s}_{o}\right) e^{-\kappa_{\lambda}\left(s-s_{o}\right)} d \lambda
$$

where $M_{\lambda}\left(\vec{s}_{o}\right)\left(W \cdot m^{-2} \cdot \mu m^{-1}\right)$ is the incident spectral emissive power (for example, from the lamps to the polymer part). $M_{\lambda}\left(\vec{s}_{o}\right)$ is given by the Planck's law [MOD 03] and represents the power received by the skin/surface of the part from the lamp.

\subsubsection{Ray tracing method}

Different numerical methods may be used to compute the source term $\vec{\nabla} \cdot \vec{q}$. The most efficient are the ray tracing [COS 11] and Monte Carlo [HOW 98] methods. The ray tracing method is commonly used in $3 \mathrm{D}$ image rendering. This method is very close to the physics of light propagation, as a ray can represent the path of a photon (or a packet of photons).

Ray tracing allows for the simulation of a wide variety of optical effects, such as reflection, refraction and absorption. In addition, this method enables us to take into account most of constitutive elements of an IR oven such as multiple lamps (various geometries) and reflectors (ceramic or metallic). The general principle of the method is to discretize, into a set of rays, the radiative heat flux emitted by each lamp. Then, each ray is followed inside the oven, from its emission point and throughout the thickness of the polymer. The ray tracer computes the optical path of each ray, accounting for specular or diffuse reflections, refractions, etc.

In addition, assumptions should be made for the different optical properties of lamps, reflectors and part. For example, the tungsten filament of a halogen lamp can be assumed to be a Lambertian emitter. This assumption provides the definition of ray direction vectors (Figure 13.14) for rays coming from the filament.

The direction vector is defined by two parameters: $\theta$ and $\varphi$, respectively, defined in the ranges $\left[0, \frac{\pi}{2}\right]$ and $[0,2 \pi]$. Notations are illustrated in Figure 13.14. The computation of $\theta$ and $\varphi$ has a strong effect on the ray tracing accuracy. Determinist discretization of the emission space could lead to errors due to the ray effect. Computing the two angles by stochastic variables avoids this [LI 97]. Another advantage of the stochastic method is that it is also possible to control the convergence of the computation. In addition, the direction change of a ray that 
polymer-air or matrix-fiber interface (for composite) is given by the Snell-Descartes law (Figure 13.15):

$$
n_{1} \sin \theta_{1}=n_{2} \sin \theta_{2}
$$

where $n_{1}, n_{2}$ are, respectively, the refractive indices of mediums 1 and 2 .

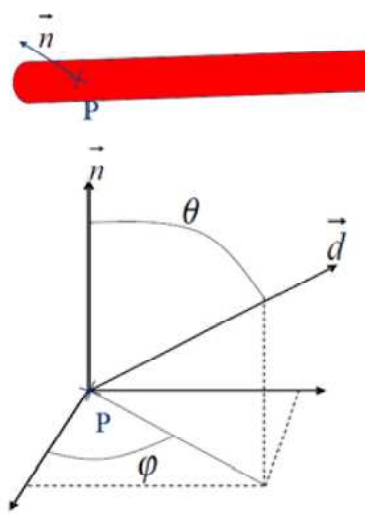

Ray direction definition

(a)

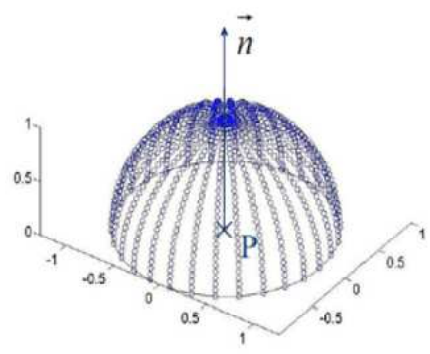

Lambertian emissivity

(b)

Figure 13.14. Ray definition for ray tracing [COS 11]

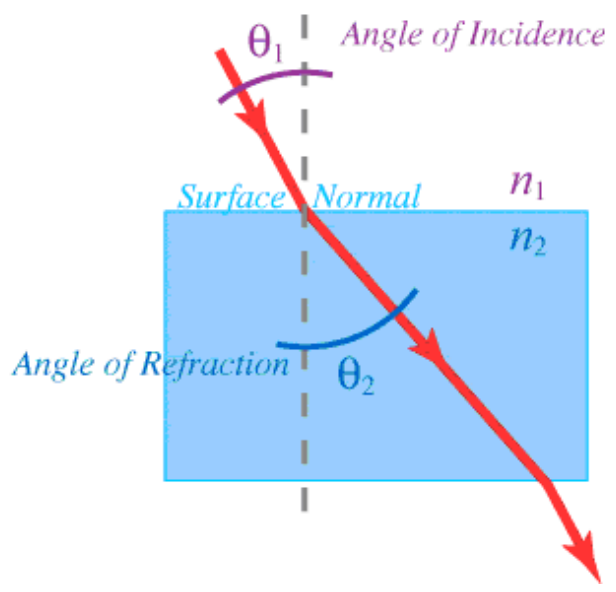

Figure 13.15. Ray refraction at interface crossing. For a color version of the figure, see www.iste.co.uk/boyard/heat.zip 
As a result, the ray tracing method can be time-consuming. It is therefore very important to optimize the number of rays as well the stochastic computation of polar emissions [COS 11].

\subsection{Polymer processing applications}

There are many applications of infrared heating in polymer processing (see section 13.1). In this section, we will focus on two promising approaches: one dealing with IR heating of thermoplastic material, "Optimization of preform temperature for ISBM process" (this study was conducted within the framework of 6th EEC framework. STREP project APT_PACK; NMP-PRIORITY) and the other one with thermoset curing "Optimal infrared composite curing" (this research work was supported by TOSHIBA LIGHTING company).

\subsubsection{Optimization of preform temperature for the ISBM process}

As described previously, the IR heating step is crucial for bottle forming. The important question is: what is the optimal preform temperature distribution before inflating? Answering this question is not easy because the main interest of the manufacturer tends to the quality of the final product (thickness distribution, mechanical properties, bottle transparency, etc.). Thus, if we define the problem in terms of an optimization algorithm, we understand that the relevant objective function is related to the forming step (for example, bottle thickness, top-load, etc.) while the optimization variable (temperature) deals with the IR heating step. This means that coupling between both steps (heating and forming) is necessary. In order to reduce CPU time, we have decided to split the optimization problem into two steps:

- First, using an interpolation function of the temperature versus length of the preform, we have computed the optimal values of temperatures located at different heights of the preform due to blow-molding numerical simulations. Full details of the procedure are given in [BOR 09]. The objective function is defined as the standard deviation of the computed bottle thicknesses. The preform geometry was a $18.5 \mathrm{~g}$ preform-PET T74F9 $(\mathrm{IV}=0.74)$. The in-lab blowing machine including an IR oven and a $50 \mathrm{cl}$ bottle mold is represented in Figure 13.16.

Figure 13.17 illustrates the temperature distribution along the preform length before and after optimization. Initial conditions were chosen in order to apply a uniform temperature on the preform. In addition, the optimal temperature distribution determined by the industrial partner (Logoplast Company) is plotted. Note that there is a good agreement in the trends between the temperature profile 
experimentally determined (trial-and-error method) within industrial conditions, and the temperature distribution computed using the optimization method.

The uniform temperature distribution leads to a strongly non-uniform thickness distribution for the bottle, as illustrated by Figure 13.18. After optimization, there is a temperature gradient along the preform length, which provides a more uniform thickness.

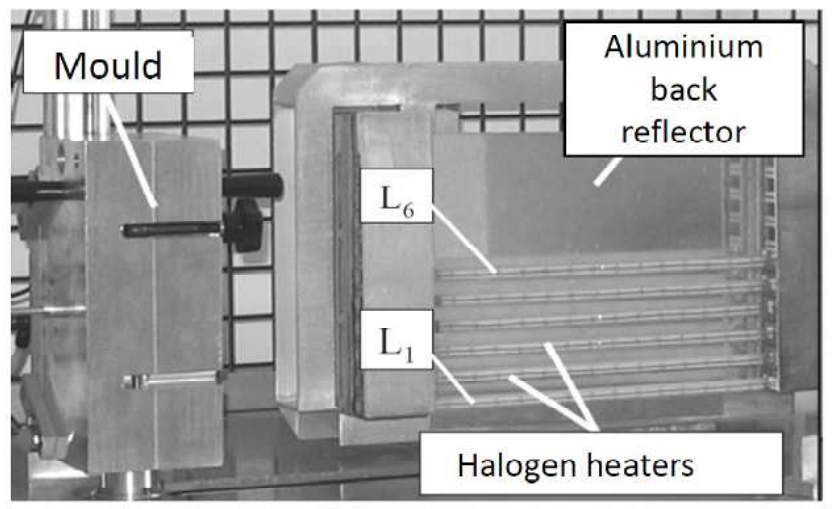

Figure 13.16. In-lab blow molding machine

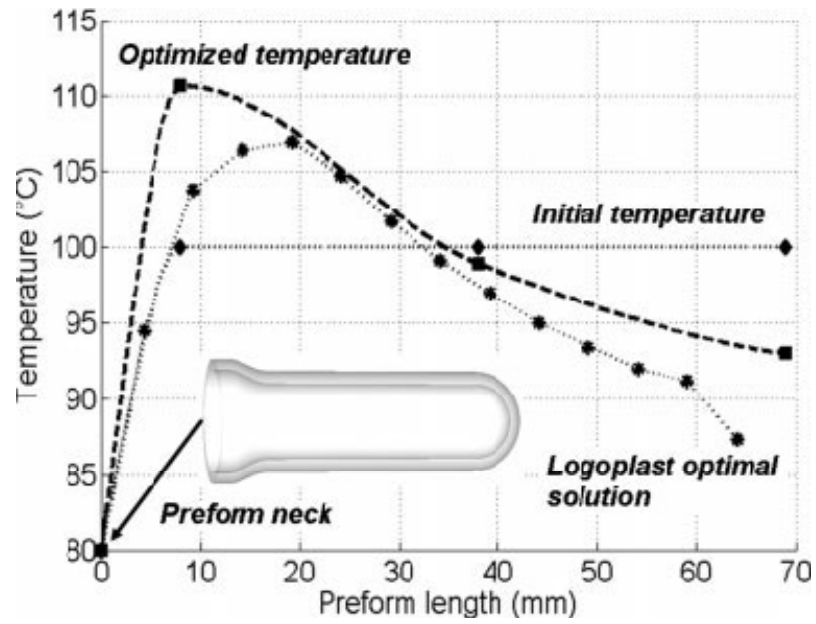

Figure 13.17. Initial and optimized temperature distributions along the preform length 


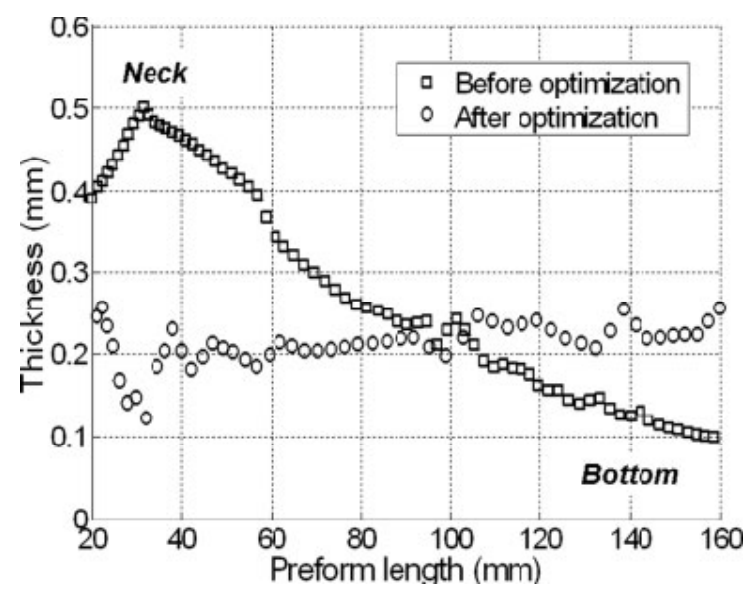

Figure 13.18. Thickness distribution of the bottle before and after optimization

- Then, when the optimal temperature distribution has been computed, a second optimization procedure is applied for computing the IR oven optimal parameters. The semi-transparent infrared model has been described in section 13.3. It is important to note that, in order to account for preform rotation, the source term has to be computed as a periodic function versus time in the numerical model. Figure 13.19 shows an example of periodic source term, plotted versus time and distributed inside the preform mesh (Figure 13.20). The computations have been performed using ray tracing in-lab software (so called RAYHEAT) and then coupled to commercial FEM software such as ABAQUS and COMSOL Multiphysics ${ }^{\circledR}$.

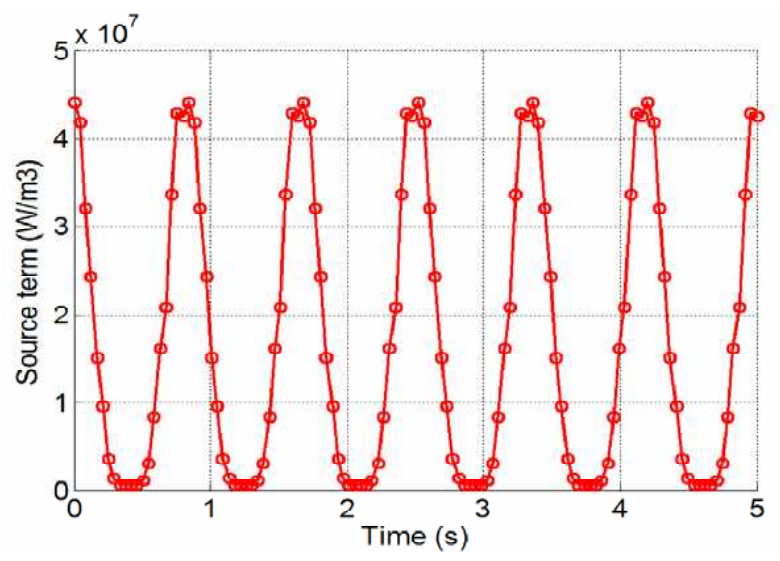

Figure 13.19. Periodic source term versus time 


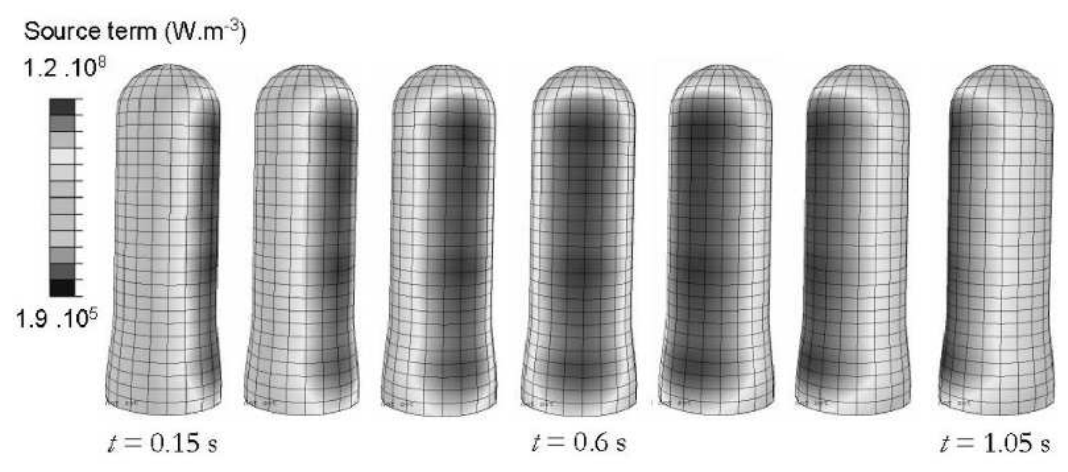

Figure 13.20. Source term distribution inside preform during time. For a color version of the figure, see www.iste.co.uk/boyard/heat.zip

In order to illustrate the accuracy of the IR heating model, a comparison is proposed between PET preform simulations and IR camera measurement (Agema $880[8-12] \mu \mathrm{m})$. Six halogen lamps of Philips $2 \mathrm{~kW}$ are set to power percentages referenced in Table 13.6.

\begin{tabular}{|c|c|c|c|c|}
\hline$P_{1}(\%)$ & $P_{2}(\%)$ & $P_{3}(\%)$ & $P_{4}(\%)$ & $P_{5}(\%)$ \\
\hline 100 & 100 & 20 & 5 & 60 \\
\hline
\end{tabular}

Table 13.6. Percentage power of each halogen emitter

In addition, the distance between the lamps and the preform, as well as the mesh for the heaters and preform, is represented in Figure 13.21. For this case, the rotation speed of preform is $1.1 \mathrm{~s}^{-1}$. The heating time is $25 \mathrm{~s}$ while cooling time is $10 \mathrm{~s}$. For the polar emission of each lamp, 1.5 million rays have been used. CPU time for computation of source term is $90 \mathrm{~min}$ (T9500 $2.6 \mathrm{GHz}, 3 \mathrm{Go} \mathrm{RAM}$ ). In addition, the preform is meshed with 6,000 tetrahedral nodes (20 hoop nodes, 10 thickness nodes and 30 height nodes). Figure 13.22 shows the excellent agreement between computed temperature versus preform height and experimental data at the end of heating time for the outside preform surface temperature. The average relative error is $1.7 \%$. 


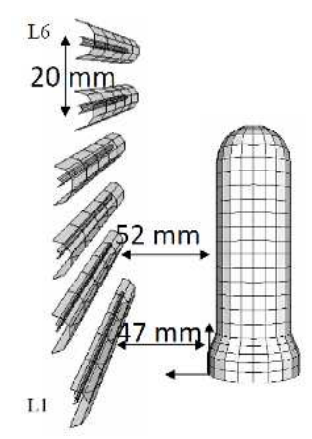

Figure 13.21. Geometrical definition of oven

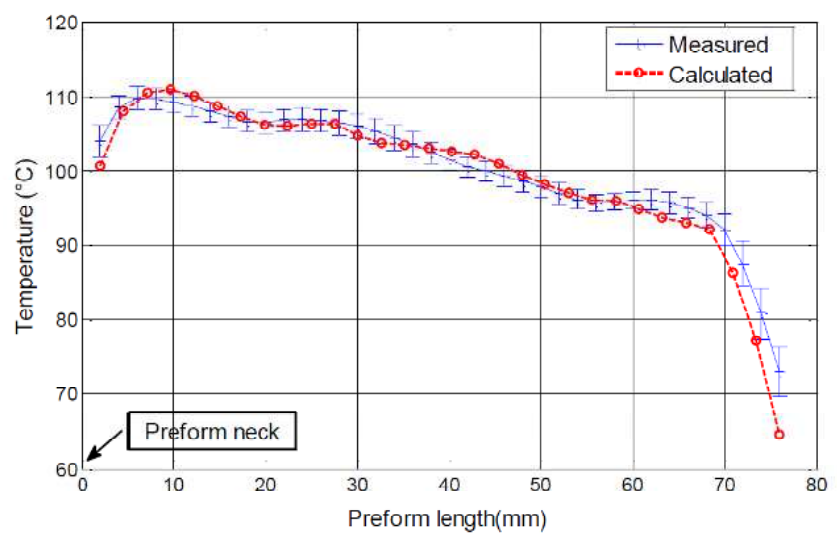

Figure 13.22. Comparison between computed and experimental temperatures versus height. For a color version of the figure, see www.iste.co.uk/boyard/heat.zip

Now, using the optimal temperature profile calculated in the previous step (Figure 13.17), we can perform an optimization procedure using the nonlinear constrained algorithm sequential quadratic programming (SQP) in order to calculate the best heating system parameters [BOR 07]. The objective was to obtain the desired temperature along the preform length, by modifying the process parameters related to the IR oven. In the case presented, seven optimization variables associated with the percentage of power of lamps of an industrial oven (Logoplast Company) have been used. The objective function $\mathrm{f}$ is simply defined as follows:

$$
f(X)=\sum_{i=1}^{7}\left(T_{\text {comp }, i}(X)-T_{o b j, i}\right)^{2}
$$


where $\mathrm{X}$ represents the set of optimization variables, $T_{\text {comp }, i}(X)$ is the computed temperature for the ith lamp and $T_{o b j, i}$ is the desired temperature value for the ith lamp. The temperature of each lamp can be related to the lamp power using the Stefan-Boltzmann law (section 13.2.2). Starting with the initial values referenced in Table 13.7, we obtain after four optimization iterations and a CPU time of $7 \mathrm{~h} 30$ min on a Pentium M 1.8 Ghz 512 Mo, the optimized values.

\begin{tabular}{|c|c|c|c|c|c|c|c|c|}
\hline & $\mathrm{P}_{1}(\%)$ & $\mathrm{P}_{2}(\%)$ & $\mathrm{P}_{3}(\%)$ & $\mathrm{P}_{4}(\%)$ & $\mathrm{P}_{5}(\%)$ & $\mathrm{P}_{6}(\%)$ & $\mathrm{P}_{7}(\%)$ & $\mathrm{f}\left({ }^{\circ} \mathrm{C}^{2}\right)$ \\
\hline $\begin{array}{c}\text { Initial } \\
\text { value }\end{array}$ & 35 & 35 & 35 & 35 & 35 & 35 & 35 & 0.134 \\
\hline $\begin{array}{c}\text { Optimized } \\
\text { value }\end{array}$ & 39.1 & 35.9 & 17.2 & 19.2 & 23.4 & 29.3 & 33.1 & 0.016 \\
\hline
\end{tabular}

Table 13.7. Initial and optimized values of lamps power

Figure 13.23 shows the comparison between the temperature profiles before and after optimization. The obtained profile fits well the targeted profile.

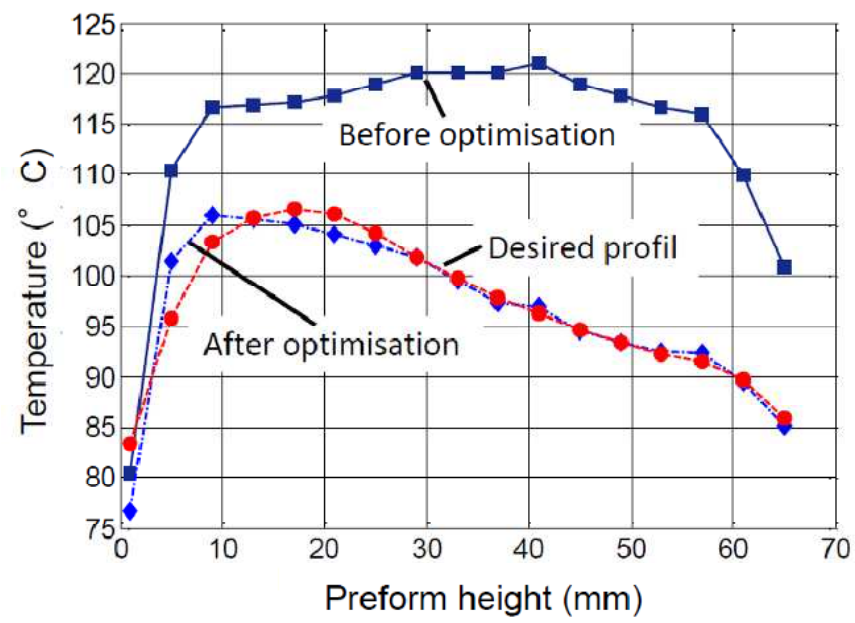

Figure 13.23. Temperature profiles before and after optimization. For a color version of the figure, see www.iste.co.uk/boyard/heat.zip

Other parameters such as heating time and cooling time can be added as optimization variables. We may also improve the objective function by accounting for energy efficiency for example. 
a)

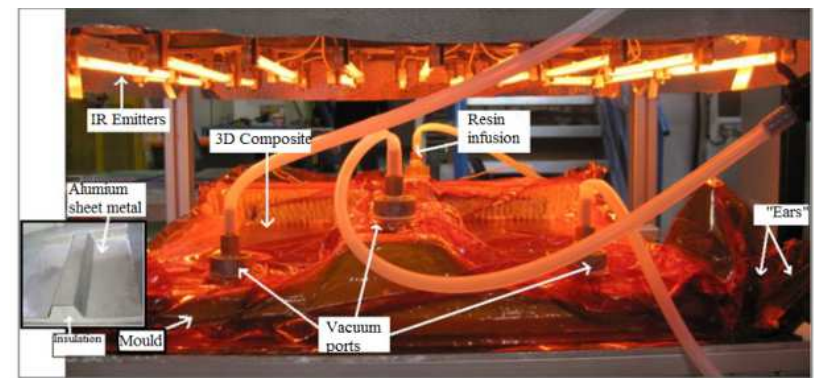

b)
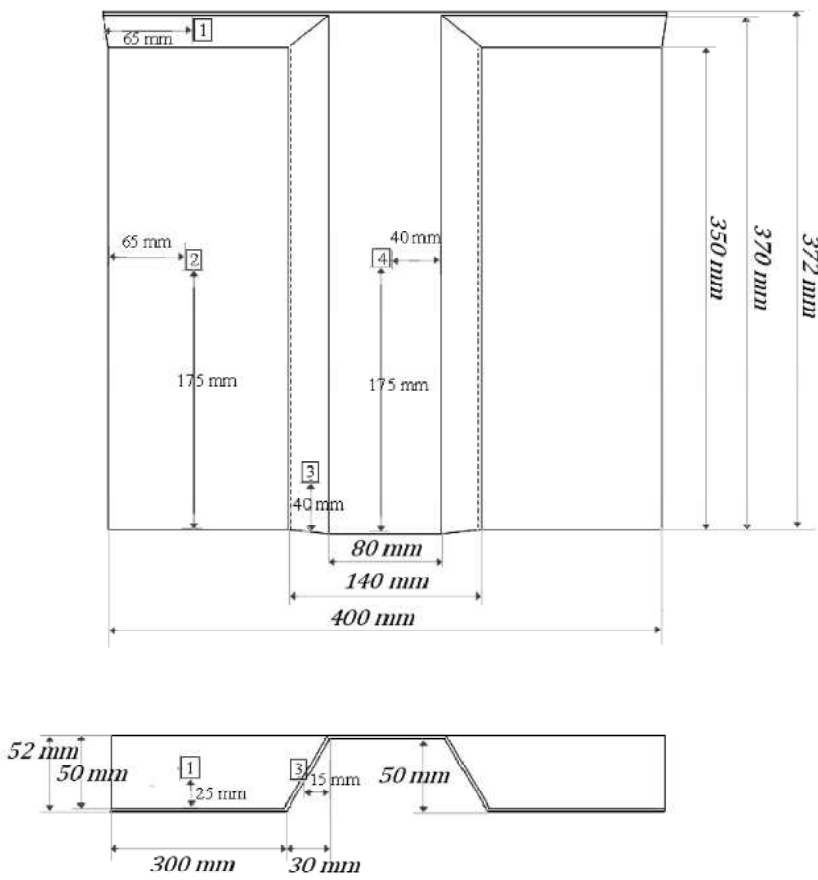

Figure 13.24. Infrared composite curing process

\subsubsection{Optimal infrared composite curing}

Epoxy resins have several applications in the aerospace and automobile industry. Because of their good adhesive properties, superior mechanical, chemical and thermal properties and resistance to fatigue and microcracking, they produce highperformance composites. In addition, rising energy costs have also promoted an interest in using infrared energy to manufacture these composites [KAR 00, CHE 02]. In the technology presented here, the composite is cured using an IR oven which includes halogen lamps (Figure 13.24(a)). The liquid resin infusion (LRI) 
process is used to manufacture the composite, whereby liquid resin (epoxy resin: RTM6) is infused through a fiber reinforcement (carbon reinforcement IMS60, non crimp fabric [45/90/-45/0]) previously in a one-sided mold (Figure 13.24(a)). The 3D geometry used to validate numerical simulations is a simplified car underbody and its dimensions are sketched in Figure 13.24(b). The manufacturing temperature for such composite is between 160 and $190^{\circ} \mathrm{C}$.

The objective of this work was to compare the temperature measurements of the 3D composite geometry realized using an infrared camera and thin thermocouples (less than $100 \mu \mathrm{m}$ in diameter) versus numerical simulations.

In order to measure surface temperatures, we used a microbolometer camera equipped with a lens of focal length $F=18 \mathrm{~mm}$. Its resolution is $320 \times 240$ pixels. Its spectral range corresponds to the average infrared $(7.3-13 \mu \mathrm{m})$. The image frequency is $60 \mathrm{~Hz}$. The apparent emissivity of the composite in the spectral range of the camera $\mathcal{E}_{7-13}=0.95$ has been calculated due to the apparent reflectivity $R_{7-13}$ [SIE 02]. The apparent reflectivity has been deduced from spectral reflectivity measurements, performed due to an infrared spectrometer Bruker Vertex 70. It is to be noted that this spectrometer is equipped with two detectors enabling coverage of all the IR spectral ranges $(0.8$ to $25 \mu \mathrm{m})$. An important detail is that the resulting measurement includes all the different films used for resin infusion (recovery surface and demolding fabric) as well as carbon fibers.

In the previous papers [NAK 13, NAK 11], a FE thermal model based upon the COMSOL Multiphysics ${ }^{\mathrm{TM}}$ software was fully detailed, for the prediction of the infrared incident heat flux on the top surface of the composite during heating. Although we have applied the ray tracing method previously [NAK 10], it is not convenient to use it for an industrial application because of the computational expense when solving the radiation problems and analysis time resulting from coupling ray tracing method with the optimization algorithms. The method employed by the FE heat transfer solver to compute the radiation heat transfer between opaque diffuse surfaces is based upon radiosity which is defined as the total radiative flux leaving a surface (see section 13.3.1).

The composite was meshed into 8,248 tetrahedral elements. A very dense mesh is used for the volume material discretization along and near the edges highlighted in Figure 13.25(a), as higher temperature gradients will occur here, while a coarser mesh is used in the remaining volume. The resulting temperature distribution is represented in Figure 13.25(b). Due to the optimization procedure [NAK 10], the maximum temperature difference throughout the composite geometry is less than 
$5^{\circ} \mathrm{C}$. Again, the positions and associated numbers of thermocouples and surfaces are sketched in Figure 13.25(b).

a)

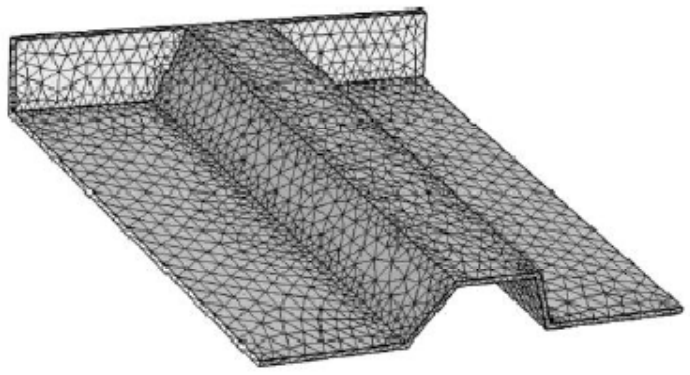

b)

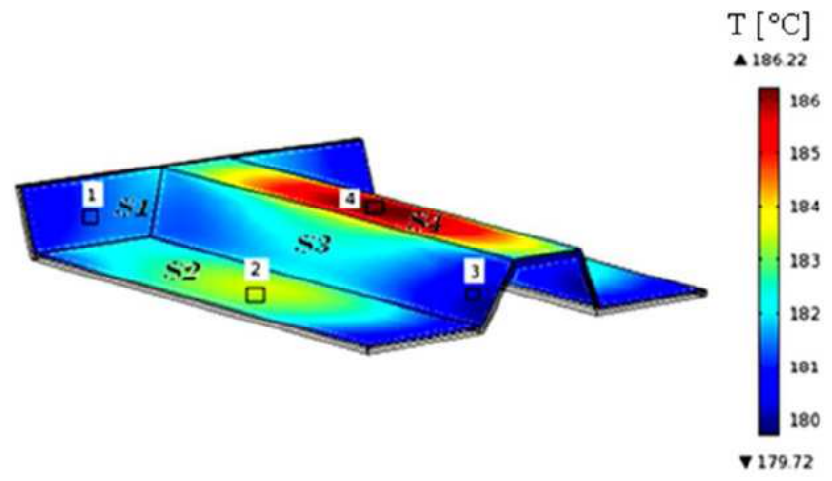

Figure 13.25. 3D mesh of the composite geometry and computed temperature distribution. For a color version of the figure, see www.iste.co.uk/boyard/heat.zip

In Figure 13.26, measured temperatures issued from both thermocouples and an IR camera, and computed ones, are plotted versus time. The 3D numerical model is developed in the finite element software COMSOL Multiphysics ${ }^{\mathrm{TM}}$, where the heat balance equation is coupled with the cure kinetic model of the resin [SIE 02]. The computed radiative heat flux is exported to COMSOL Multiphysics ${ }^{\mathrm{TM}}$, and imposed as a boundary condition on the top surface of the composite. This numerical model allows the calculation of the temperature distribution in the composite during curing as well as the degree of curing. From a global point of view, the agreement is unexpectedly good considering all the input data involved in the numerical model. At position 1, the measured temperature using thermocouple (TC1) and computed 
one is in good agreement, especially during the temperature rise. However, a temperature difference of $5^{\circ} \mathrm{C}$ is calculated during the steady state.

At position 2, the surface temperature measured by IR camera is slightly higher than those measured by thermocouple (TC2). Nevertheless, the measured and computed temperatures exhibit again a good agreement.

At position 3, again the best agreement between experimental and numerical temperatures occurs during temperature rise (transient state). A temperature difference of $8^{\circ} \mathrm{C}$ is observed during the steady state, both with IR camera and thermocouple measurement. Indeed, the proximity of this thermocouple to a vacuum port leads to underestimate the measured temperature.
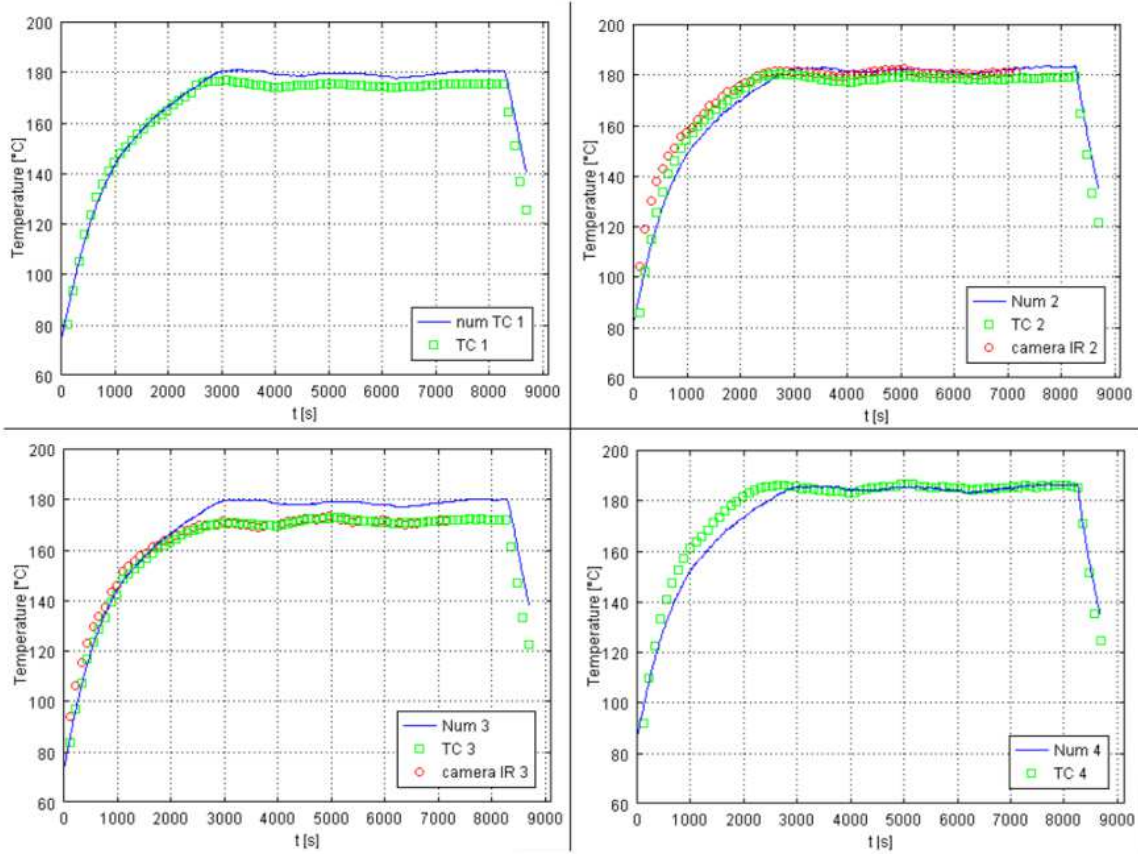

Figure 13.26. Comparison between measured and computed temperatures. For a color version of the figure, see www.iste.co.uk/boyard/heat.zip

Different temperature measurements due to an IR camera and thermocouples allows for validating computed temperatures. In order to simulate the curing 
process, 3D radiation numerical simulations also include a kinetic model based upon modified Kamal-Sourour equation [NAK 13, RYA 79]. For estimating the influence of IR radiation upon mechanical properties of the final composite piece, it would be important to achieve mechanical tests (DMA, 3-points bending, shear test, etc.). For example, comparisons may be performed between samples cured by conduction mode (or autoclave), and by infrared oven. In addition, careful examinations of SEM images would lead to better understand the microstructure of the composite piece obtained using the IR curing process.

\subsection{Future work}

Infrared heating of polymers is still a challenging and exciting research topic. Many questions still remain unanswered. An important one deals with the interaction of radiation and the microstructure of polymer. As mentioned previously (see section 13.3.2.2), when adding submicronic mineral fillers or dyes in PET [BIL 12], IR heating is affected. This means rays may be refracted and radiation diffusion may occur. This is also the case for thermoplastic composites [AKU 15] (for example, polycarbonate matrix and glass fibers reinforcement) or some polymers crystallizing during IR heating. The ray tracing method can be used to account for scattering but it can be time-consuming for industrial applications. Different physical models, based on ratio between the diameter $\mathrm{D}$ of spherical particles and IR wavelength $\lambda$ (size parameter known as $\pi \mathrm{D} / \lambda$ ), such as Rayleigh approximation and Mie theory [VAN 81] may be applied to account for interactions between IR radiation and microstructure as in [DEN 96].

Another interesting challenge deals with the measurement of the absorption coefficient for semi-transparent polymers (for example, PET). The common procedure uses a spectrometer in transmission mode. But measuring the absorptivity independently of the thickness samples requires careful cuts using microtome, meaning operator dependancy. In the case of specular reflection, we could take advantage of Kramers-Kronig [KIT 04] relation in order to assess to the absorption coefficient.

In addition, the characterization of heater lamps and more particularly halogen lamps, still remains a big challenge. Most laboratories are not equipped for measuring such high temperatures ( $\cong 2500 \mathrm{~K})$. Again, an IR camera may be a good way of evaluating the desired temperature. The experimental set-up consists of heating a thermoplastic sheet for which all the thermo-physical and thermo-optical properties are well known. Thus, when performing accurate numerical simulations of infrared heating, we can apply an inverse design approach in order to find the 
optimal temperature value. The objective function may compare, for example, experimental and numerical temperature profiles at the back surface of the polymer sheet. For this method, work has to be done on the influence of the boundary condition such as convection and environment temperature. Lastly, the experiments involving an infrared camera could also bring information on the irradiance map in $\mathrm{W} / \mathrm{m}^{2}$ provided by the halogen lamp on the thermoplastic sheet (profile, amplitude, etc.).

\subsection{Acknowledgements}

The authors warmly thank all the $\mathrm{PhD}$ and postdoctoral students for their indispensable help, exciting scientific discussions and apologize to those they forgot to mention:

Serge Monteix, Sylvia Andrieu, Cedric Champin, Maxime Bordival, Benoit Cosson, Sawsane Nakouzi, Johann Pancrace, Myriam Dauphin, Andre Chateau and Akue Asseko, among others.

Thanks also go to our colleague Jean-Paul Arcens, now retired, who helped us in many technical aspects during the past decade on the different problems described in this chapter.

Special thanks go to our colleague Gary Menary (Queen's University of Belfast) for reviewing this chapter.

A special thought for our colleague Bernard Plantamura (Sidel Company) who left us prematurely in 2015 .

\subsection{Bibliography}

[ADE 02] ADEME, "Energies radiantes et leurs applications industrielles", available at: http://www.ademe.fr/energies-radiantes-applications- industrielles, 2002.

[AKU 15] AKUE Asseko A.-C., Cosson B., SCHMidt F. et al., "Laser transmission welding of composites-Part A: thermo-physical and optical characterization of materials", Infrared Physics and Technology, vol. 73, pp. 304-311, 2015.

[AND 05] ANDRIEU S., Étude expérimentale et numérique du chauffage infrarouge court sur des plaques polymères pour le thermoformag, PhD Thesis, ENSMP, 2005. 
[BAR 94] BARDON J.P., JAVELAS R., Mesure de températures de surface sur des matériaux semi-transparents, Internal report, CNRS-Ecotech/Ademe-Arc-Metrologie, 1994.

[BIL 12] Billon N., Haudin J.M., VAllot C. et al., "Stretch blow molding of mineral filled PET”, Key Engineering Materials, vol. 504-506, pp. 1099-1104, 2012.

[BOR 07] Bordival M., Schmidt F., LE MAOUlt Y., "Numerical modelling and optimization of infrared heating system for the blow molding process", Proceedings of 9th Esaform Conference on Material Forming, 2007.

[BOR 09] Bordival M., Le MaOult Y., Schmidt F., "Optimization of preform temperature distribution for the stretch-blow moulding of PET bottles: infrared heating and blowing modeling", Polymer Engineering and Science, vol. 49, no. 4, pp. 783-793, 2009.

[CHE 02] Chern B.-C., MoOn T.J., Howell J.R., "On-line processing of unidirectional fiber composites using radiative heating: I. model”, Journal of Composite Materials, vol. 1935, pp. 1905-1934, 2002.

[COH 85] Cohen M.F., Greenberg D.P., "The hemicube: a radiosity approach for complex environments”, Computer Graphics, vol. 19, no. 3, pp. 31-40, 1985.

[COS 11] Cosson B., Schmidt F., Le MaOult Y. et al., "Infrared heating stage simulation of semi-transparent media (PET) using ray tracing method", International Journal of Material Forming, vol. 4, pp. 1-10, 2011.

[DEN 96] Denis A., Dargent E., Lebaudy P. et al., "Dependence on the spectral scattering coefficient on cristallinity into semicrystalline polyester", Journal of Applied Polymer Science, vol. 62, pp. 1211-1218, 1996.

[FRA 02] Franck I., DAVID D.W., Fundamentals of Heat and Mass Transfer, 5th ed., John Wiley \& Sons, 2002.

[HAI 94] HaiJ N., Spruiell J.E., "Radiation pyrometry on semi-transparent media with wavelength dependent absorption coefficient", Polymer Engineering and Science, vol. 34, no. 2, pp. 122-127, 1994.

[HOW 98] Howell J.R., "The Monte-Carlo method in radiative heat transfer", Journal of Heat Transfer, vol. 120, pp. 547-560, 1998.

[IEC 87] IEC, International electrotechnical vocabulary, Lighting, Internal Report, 1987.

[KAR 00] Karkanas P.-I., PARTRIDGe I.-K., "Cure modelling and monitoring of epoxy/amine resin systems II. Network formation and chemoviscosity modeling", Journal of applied Polymer Science, vol. 77, pp. 2178-2188, 2000.

[KIT 04] KitTel C., Introduction to Solid State Physics, 8th ed., Wiley, 2004. 
[KNI 96] KNIGHTS M., “The truth about heaters", Plastics Technology, vol. 42, no. 5, pp. 3846, 1996.

[LI 97] Li B., TAO W., LiU R., "Ray effect in ray tracing method for radiative heat transfer", International Journal of Heat Mass Transfer, vol. 40, pp. 3419-3426, 1997.

[MAO 05] LE MAOUlT Y., Mesures infrarouges appliquées aux transferts thermiques et à la science des matériaux, confrontation de modèles aux expérimentations, Thesis, Mines Albi / INP Toulouse, 2005.

[MOD 03] Modest M.F., Radiative Heat Transfer, 2nd ed., Acad. Press, New York, 2003.

[MON 01] MonTEIX S., Modélisation du chauffage convecto-radiatif de préformes en P.E.T. pour la réalisation de corps creux, PhD Thesis, ENSMP, 2001.

[MON 04] Monteix S., Le MaOult Y., Schmidt F. et al., "Quantitative infrared thermography applied to blow moulding process: measurement of a heat transfer coefficient”, Quantitative Infrared Thermography Journal, vol. 1, no. 2, pp. 133-150, 2004.

[NAK 10] Nakouzi S., Pancrace J., Schmidt F. et al., "Curing simulation of composites coupled with infrared heating", International Journal of Material Forming, vol. 3, no. 1, pp. 587-590, 2010.

[NAK 11] NAKouZi S., PANCrace J., Schmidt F. et al., "Simulations of an infrared composite curing process", Advanced Engineering Materials, vol. 13, no. 7, pp. 604-608, 2011.

[NAK 12] Nakouzi S., Berthet F., Delaunay D. et al., "Optimization of the incident IR heat flux upon a 3D geometry composite part (carbon/epoxy)", Key Engineering Materials, vol. 504-506, pp. 1085-1090, 2012.

[NIX 15] NIXON J., Analysis of the stretch blow moulding process and subsequent simulation development, PhD Thesis, Queen's University Belfast, 2015.

[NAK 13] Nakouzi S., Berthet F., Le Maoult Y. et al., "Simulations of an infrared composite curing process", Key Engineering Materials, vol. 554-557, pp. 1517-1522, 2013.

[ROS 04] Rosato D.V., Rosato A.V., DiMattia D.P., Blow Molding Handbook, 2nd ed., Hanser Verlag, 2004.

[RYA 79] RYAN M.E., DutTA A., "Kinetics of epoxy cure: a rapid technique for kinetic parameter estimation”, Polymer, vol. 20, pp. 203-206, 1979.

[SCH 03] SchmidT F., LE MaOUlt Y., et al., "Modelling of infrared heating of thermoplastic sheet used in thermoforming process", Journal of Materials Processing Technology, vol. 143 , pp. $225-231,2003$.

[SIE 02] Siegel R., Howell J.R., Thermal Radiation Heat Transfer, 4th ed., Taylor and Francis-Hemisphere, Washington, 2002. 
[THR 99] ThrONE J.L., Understanding Thermoforming, Hanser Gardner Publications, 1999.

[VAN 81] VAN DE Hulst H.C., Light Scattering By Small Particles, Dover, 1981.

[WIL 14] Wilson C., McGranaghan G., "Infrared heating comes of age", Reinforced Plastics, vol. 58, no. 2, pp. 43-47, 2014.

[WOL 89] WoLfE W.L., Zissis G.J., The Infrared Handbook, ERIM ed., 1989. 